Ann. Sci. forest., 1979, 36 (2), 93-124.

\title{
Une étude phyło-écologique sur les plateaux calcaires du Nord-Est (Massif de Haye-54).
}

\author{
Utilisation de l'analyse des correspondances dans la typologie \\ des stations. Relations avec la productivité et la qualité \\ du hêtre et du chêne
}

\author{
M. BECKER \\ avec la collaboration technique de R. F. CLAUDE et R. SCHIPFER \\ Laboratoire de Phyto-écologie forestière, \\ Centre national de Recherches forestières, I.N.R.A., \\ Champenoux, 54280 Seichamps
}

\begin{abstract}
Résumé
Le massif étudiè $(12000 \mathrm{ha})$ est représentatif des très importantes surfaces forestières situées sur les plateaux calcaires du Nord-Est de la France. Près de 800 relevés floristiques y ont été faits, complétés par quelques observations dendrométriques ef technologiques sur les principales essences (Hêtre ef Chêne).

Une analyse factorielle des correspondances a mis en évidence l'importance décroissante, quant à la composition floristique, d'un certain nombre de facteurs écologiques - édaphiques ou climatiques - : $\mathrm{pH}$, lui même lié à l'alimentation en cau, nutrition minérale, exposition, nutrition azotée... : mais aussi l'importance non négligeable du facteur traitement sylvicole (futaie ou taillis-sous-futaie). L'analyse facilite aussi considérablement l'élaboration du tableau synthètique des stations. Neuf groupes écologiques d'espèces ont été définis, ainsi que dix stations, chacune d'elle étant caractérisée par une certaine combinaison originale des groupes écologiques, ainsi que par un type édaphique donné (grâce à l'éfude de Gury, 1972), et par une situation topographique particulière. La carte de répartition de ces stations a éfé dressće. Les stations ainsi définies et, plus encore, les groupes écologiques devraient pouvoir être largement utilisables dans le reste de la région définie.

Les mesures de hauteur dominante ont montré une bonne corrélation, pour un traitement sylvicole donné, entre fertilité et types de stations, tant pour le Hêtre que pour le Chêne (fig. 3) : mais les arbres de futaie sont nettement plus hauts que ceux de taillis-sous-futaie.

Quant à la qualité des bois, le couple de torsion (technique Polge, Keller, 1970) est apparu plus discriminant que la densité des échantillons prélevés à la tarière de Pressler. Son déterminisme se révèle plus complexe : l'espèce, la station et la structure du peuplement jouent simultanément (fig. 4 et 5). Pour l'effet-espèce, il apparaît en particulier indispensable de séparer Chênes sessile et pédonculé : pour l'effet-station, il se confirme une corrélation négative entre qualité du bois et qualité de la nutrition ; pour l'effet-structure, les houppiers développés apparaissent également plus favorables.
\end{abstract}

\section{1. - Introduction et objectifs}

L'ensemble du travail présenté ici se fixait à l'origine un triple objectif : d'amélioration de méthodes, de connaissance scientifique et d'étude concrète d'un cas intéressant pour le forestier praticien. 
Sur le plan des méthodes, il s'agissait d'apporter une nouvelle contribution à la mise au point d'un ensemble de techniques permettant l'étude des stations forestières (identification et caractérisation phyto-écologique) de la façon la plus rapide et la plus objective possible. Ces deux impératifs nous avaient, dès nos premiers travaux, orienté vers les possibilités nouvelles des outils de l'informatique (Aussenac, Becker, 1968 ; Becker, 1969). Un des objectifs de l'étude présentée est de contribuer à l'appréciation des possibilités d'une méthode d'analyse multivariable de plus en plus utilisée dans de nombreux domaines (y compris la phytosociologie) : l'analyse factorielle des correspondances.

Sur le plan des connaissances scientifiques, les objectifs étaient, d'une part d'approfondir la valeur indicatrice des espèces de la flore forestière et des groupes écologiques qui la constituent, d'autre part d'étudier les relations existant entre les stations que cette flore contribue à mettre en évidence et certaines données se rapportant aux essences forestières cultivées, en particulier leur productivité et leur qualité technologique. Concernant le premier point, l'éfude pédologique déjà réalisée par Gury (1972) était un atout considérable.

Sur un plan plus pratique enfin, il paraissait utile de s'intéresser à ce vaste massif forestier de Haye, aux portes d'une grande agglomération urbaine, oủ l'on doit de plus en plus s'efforcer de concilier des impératifs de production et d'accueil du public. Les résultats obtenus peuvent y contribuer. Ils doivent de plus pouvoir être, dans une large mesure (à préciser), extrapolés à la vaste région forestière que constituent les «Côtes de Moselle », de Neufchâteau à Briey.

\section{2. - Quelques généralités sur le massif de Haye}

Nous passerons très rapidement sur la présentation générale du massif, qui a déjà été faite par ailleurs de façon très approfondie, tant sur le plan physique que biotique (J. Venet, 1975).

Le massif de Haye forme un ensemble forestier d'environ $12000 \mathrm{ha}$, dont l'individualité au sein des Côtes de Moselle est due essentiellement aux importantes vallées qui l'enserrent : la boucle de la Moselle surtout (sud, ovest ef nord) ef la Meurthe (nord-est). Sur ce total, on compte : 7000 ha de forêt domaniale, 3500 ha de forêts communales et 1500 ha de forêt privée.

Le massif occupe un plateau légèrement inclinế vers l'ouest (altitude $380 \mathrm{à} 420 \mathrm{~m}$ ), retombant en falaise à l'est, sur la plaine liasique ; la Moselle est à 200-220 m d'altitude. Ce plateau est profondément entaillé par des vallons secs (les « fonds »), comme souvent sur plateau calcaire, qui créent ainsi une variabilité topographique importante pour l'écologie du massif (microclimats, sols).

Sur le plan géologique, les roches mères sont essentiellement constituées par les calcaires du Bajocien, reposant sur un socle de terrains liasiques (Aalénien, Toarcien et Charmouthien). Vers l'ouest, ils sont par place recouveris par le Bathonien inférieur, constitué de calcaires oolithiques terreux avec passées irrégulières de marnes. Le Bajocien (inférieur et supérieur) comprend essentiellement des calcaires oolithiques blancs compacts et quelques calcaires à entroques. 
II est indispensable de tenir compte également des formations superficielles, qui ont une importance considérable du point de vue édaphique. Ce sont, d'une part des formations calcaires (éboulis grossiers des pentes, plus ou moins mêlés d'éléments argileux ou argilo-limoneux, ou « grouine», sorte de cailloutis calcaire à éléments très fins d'origine périglaciaire), d'autre part des formations non calcaires : «terra fusca », argile rougeâtre très ancienne provenant de la décarbonatation de la rochemère ; limons éoliens et alluvions anciennes en placages d'étendue el d'épaisseur très variables, plus ou moins mélangés par cryoturbation et solifluxion à la terra fusca sous-jacente.

Sur le plan climatique, le climat lorrain est du type semi-continental, avec extrêmes de températures marqués en hiver (janvier, moyenne de $0,9{ }^{\circ} \mathrm{C}$ ) et en été (juillet, moyenne de $18,2^{\circ} \mathrm{C}$ ). La normale de précipitations est de $737 \mathrm{~mm}$ par an. Les gelées de printemps sont souvent à craindre ; il arrive aussi que la pluviosité de mai à août soit faible ; les régénérations de Hêtre sont donc fréquemment compromises.

Sur le plan forestier, les essences spontanées les plus importantes sont le Hêtre (Fagus silvatica), largement dominant, le Chêne sessile (Quercus sessiliflora) et le Chêne pédonculé ( $Q$. pedunculata) - le Chêne pubescent (Quercus lanuginosa) existe aussi, très localisé -, l'Erable plane (Acer platanoides), le Sycomore (A. pseudoplatanus), l'Erable champêtre ( $A$. campestre), le Charme (Carpinus befulus), le Frêne (Fraxinus excelsior). Il faut aussi noter l'Orme de montagne (Ulmus scabra), le Tilleul à grandes feuilles (Tilia platyphyllos), le Tilleul à petites feuilles (T. cordata), et des « fruitiers divers » : Merisier (Prunus avium), Alisier torminal (Sorbus torminalis), Alisier blanc (S. aria), Cormier ( $S$. domestica). Les introductions de résineux sont très limitées : Pin noir d'Autriche (Pinus nigra ssp. nigricans), Epicéa (Picea abies), Sapin pectiné (Abies alba) et Sapin de Nordmann (A. nordmanniana).

Au siècle dernier, le traitement en taillis-sous-futaie était la règle générale. Celuici subsiste dans un certain nombre de séries domaniales et dans les forêts communales. Ailleurs (5 $200 \mathrm{ha}$ ) a été entreprise, depuis un siècle environ, la « conversion » en futaie régulière par vieillissement progressif du taillis-sous-futaie et ensemencement naturel ; c'est à l'heure actuelle dans les meilleures stations qu'elle est la plus avancée.

Sur le plan biotique enfin, il importe surtout de signaler l'importante pression urbaine à laquelle est soumise le massif, directement au contact d'une agglomération de 300000 habitants. Si son rôle récréatif s'impose donc, il n'est pourtant pas incompatible, bien au contraire, avec son orientation vers la production de bois de qualité supérieure (Hêtre de déroulage en particulier). Une meilleure connaissance des milieux devrait d'ailleurs largement faciliter cette harmonisation des objectifs.

L'influence du gibier (Chevreuil surtout, Sanglier) est relativement faible : quelques dégâts seulement dans les plantations, résineuses et feuillues.

\section{3. - Méthodies de travail}

\section{1. - Introduction}

Une préoccupation de base étant la possibilité d'un passage efficace à l'application pratique, les efforts doivent tendre vers un déroulement aussi standardisé que 
possible de l'essentiel des opérations : en particulier la prise des données sur le terrain et les premières phases de leur interprétation (Aussenac, Becker, 1968 ; Becker. 1969, 1972: Timbal, 1973 ; Picard, 1970, 1976).

Si la méthodologie du travail de terrain ne pose plus guère de problèmes de fond cruciaux, il n'en était pas de même, il y a peu de femps encore, en ce qui concerne le traitement statistique des données recueillies, toujours très nombreuses. Les méthodes d'analyse multivariable testées se heurtaient surtout à la taille insuffisante des ordinateurs disponibles, incapables de traiter simultanément un nombre suffisant de variables. Pour cette raison, les variables analysées furent d'abord uniquement les données écologiques décrivant le milieu physique (analyse en composantes principales). Les variables floristiques n'étaient prises en compte que dans un deuxième temps, et regroupées en fonction de leur réponse aux «facteurs » synthétiques (les composantes principales) élaborés par l'analyse précédente (Becker, 1969).

Les performances des ordinateurs augmentant, il fut possible de tester la démarche inverse, c'est-à-dire, sur un échantillon limité d'espèces (une cinquantaine), d'étudier directement leur sociabilité, en observant ensuite leurs relations avec les variables écologiques mesurées. Les éfudes comparatives menées (Becker, 1969 ; Picard, 1970) ont démontré l'efficacité des deux approches, tout en concluant pourtant à la plus grande sécurité de la seconde.

N'étant aujourd'hui pratiquement plus limité par le nombre de variables à traiter simultanément (plusieurs centaines), c'est dans cette voie qu'avec d'autres (Picard, 1976 ; Juratic et Plan, 1976), nous nous sommes engagé pour les éfudes phyto-écologiques de massifs forestiers. La méthode utilisée l'est d'ailleurs de plus en plus, et avec bonheur, chez les biologistes en général et les phytosociologues en particulier (Briane et al., 1974 ; Dumé, 1975 ; Lacoste et Roux, 1971, 1972 ; Romane, 1972).

\section{2. - La collecte des données}

\subsection{Echantillonnage des points-relevés.}

La technique de l'échantillonnage systématique à mailles carrées a été adoptée. Toutefois sa densité n'a pas été uniforme, mais «stratifiée » en fonction d'un facteur essentiel évident de variation stationnelle : la topographie. Les vallons qui entaillent le plateau n'occupent environ que $1 / 5$ de la surface totale du massif, mais leur variabilité écologique apparaissait a priori plus importante. Pour parer au risque de les sous-échantillonner, la densité des points-relevés y a été quadruplée. Cette densité forte a également été adoptée pour une forêt située à l'ouest du massif, spéciale dès l'abord par son substrat (Bathonien et alluvions profondes) et ses sols (souvent un peu hydromorphes).

La distance entre relevés a été fixée à $500 \mathrm{~m}$ sur plateau (un relevé pour $25 \mathrm{ha}$ ) et $250 \mathrm{~m}$ ailleurs (un relevé pour $6,25 \mathrm{ha}$ ). Le total des relevés ainsi pointés (huit cent vingt-quatre) reste dans des limites acceptables (sur le plan volume de travail sur le terrain), tout en permettant in fine une ébauche cartographique de la répartition des types stationnels sans retour sur place (acceptable au 1/50 000). En fait, quelques relevés ne purent être effectués pour des raisons diverses (reboisements résineux, défrichements...), et le nombre définitif s'établit à sept cent soixante-douze. 


\subsection{Nature des données inventoriées.}

Chaque relevé a fait l'objet d'un inventaire floristique classique, selon la méthode de Braun-Blanquet, sur des surfaces de 200 d $400 \mathrm{~m}^{2}$ selon l'homogénéité apparente du tapis herbacé (notion d'aire minimum). Les strates arborescente, arbustive et herbacée (englobant la strate muscinale) ont été distinguées sur le terrain ; mais strates arbustive et herbacée ont été confondues pour le traitement.

Par ailleurs, les opérations de terrain ont été singulièrement allégées grâce à l'étude pédologique et à la cartographie des sols du massif réalisées par Gury (1972), qui ont permis d'établir les rapports sol-végétation.

En ce qui concerne l'aspect proprement sylvicole, quelques observations sommaires ont été faites sur chacun des sept cent soixante-douze relevés : mesure de la hauteur et de la circonférence à 1,30 $\mathrm{m}$ de deux arbres parmi les dominants (Hêtre et/ou Chêne) ; prélèvement d'une carotte à la tarière de Pressler : mesure du « couple de torsion » nécessaire pour la pénétration de la tarière selon la technique Polge-Keller (1970).

La circonférence $C$ ef l'accroissement moyen annuel sur le rayon a permettent d'apprécier sommairement l'âge des arbres, et donc d'affecter au relevé un indice de fertilité en se rapportant à des courbes de croissance préétablies. Le couple de torsion est un indice intéressant pour une première appréciation de la qualité du bois.

\section{3. - L'interprétation des données floristiques}

\subsection{L'analyse factorielle des correspondances.}

Deux cent quarante et une espèces ont été rencontrées au cours de l'étude. Pour l'interprétation statistique, les essences forestières ont été dédoublées (strate arborescente et strate arbustive + herbacée), ce qui porte d̀ deux cent soixante-sept le nombre de variables floristiques (cf. liste en annexe).

Ces observations ont été codées, transcrites sur feuilles « quatre-vingt colonnes » et perforées.

II n'est pas question d'exposer ici dans le détail les formulations mathématiques de l'analyse factorielle des correspondances. Elles dépassent d'ailleurs assez rapidement notre compétence personnelle, et l'on se reportera plutôt à Lacoste et Roux (1972), Escoufier (1972), Romane (1972), Benzecri (1973). Mais nous pensons utile d'en rappeler très sommairement la démarche.

Les coefficients d'abondance-dominance affectés aux $n=$ deux cent soixantesept espèces dans les $p=$ sept cent soixante-douze relevés forment un tableau de données $k_{i j}$. Dans ce qui suit, espèces et relevés jouent des rôles parfaitement symétriques ef peuvent être permutés ; nous ne raisonnerons donc que sur les espèces.

II s'agit de comparer les «profils» d'apparition des diverses espèces sur l'ensemble des relevés. Mais auparavant, toutes les espèces n'ayant pas la même importance, on les ramène à un rang d'égalité en travaillant en fait sur des termes $k_{i j} / k_{i,}$, dans lesquels :

$$
k_{i}=\sum_{j=1}^{p} k_{i j}
$$

(somme des coefficients d'abondance-dominance de l'espèce $i$ dans les $p$ relevés). 
Dans l'espace à $p$ dimensions des relevés, chaque espèce $i$ a pour coordonnées $k_{11}, k_{12}, \ldots, k_{i p}$ et pour « poids $» k_{i}$. Le but est la recherche des proximités entre points, c'est-à-dire des degrés de ressemblance des distributions d'espèces dans les relevés : si deux espèces $i$ et ' $i$ ' sont proches, leurs profils se ressemblent.

L'analyse détermine au mieux les axes de plus grand allongement du nuage de points ainsi défini : ce sont les axes factoriels, qui extraient le maximum d'information sur la proximité entre espèces, c'est-à-dire fels que la projection des espèces sur eux déforme le moins possible le nuage.

De proche en proche, on élabore ainsi une série d'axes factoriels, orthogonaux (et donc indépendants deux à deux). La projection du nuage sur ces axes entraine une déformation croissante; ils sont donc de moins en moins explicatifs.

La seule projection d'une espèce sur un axe donné n'est pourtant pas suffisante pour apprécier son intérêt. II importe aussi de connaître sa distance à l'axe et, partant, son caractère plus ou moins spécifique de cet axe. A cette fin, est calculé le cosinus de l'angle fait avec l'axe par la droite joignant l'espèce au centre de gravité du nuage : plus ce cosinus est proche de 1. plus l'espèce est bien caractéristique de l'axe en question.

\subsection{Utilisation des résultats de l'analyse.}

Les divers axes extraits par l'analyse factorielle absorbent des pourcentages décroissants de la variation totale. En général, seuls les quelques premiers axes (ou facteurs) ont une signification biologique interprétable, souvent les deux ou trois premiers, parfois les cinq ou six premiers. Pour en déterminer le nombre, on admet, avec Benzecri (1973), qu' « en analyse multidimensionnelle, la complexité des informations traitées et des réponses obtenues est telle qu'un résultat issu de fluctuations aléatoires a très peu de chances d'être interprétable : par conséquent, on peut légitimement admettre que tout ce qui est interprétable est valide ».

Lorsque les données sont recueillies à l'échelle d'un massif forestier, ou d'une petite région forestière, les axes apparaissent être le reflet des grands facteurs écologiques conditionnant la répartition des plantes (alimentation en eau, en éléments minéraux, lumière...) ou, parfois, de facteurs biotiques particuliers (Picard, 1976).

L'analyse des correspondances permet donc d'abord de « comprendre» le domaine étudié, ce qui est essentiel : les facteurs de variations, par ordre d'importance ; la réaction des espèces à ces divers facteurs ; la façon dont le milieu se structure à partir d'eux.

Mais les résultats obtenus ne permettent que rarement de discerner des limites évidentes entre groupes d'espèces d'une part, groupes de relevés d'autre part. Le recours au traditionnel tableau phytosociologique nous apparait souhaitable pour déceler les limites les plus adaptées. Mais l'élaboration, souvent très fastidieuse, de ce tableau est considérablement facilitée par les résultats de l'analyse qui précède. Cette opération consiste essentiellement à « diagonaliser 》 le tableau initial des données, c'est-à-dire à disposer espèces (en lignes) et relevés (en colonnes) selon leur réponse au facteur essentiel de variation du milieu étudié. Or ce « facteur essentiel» est parfaitement décelé par l'analyse : c'est celui qui correspond à l'axe I. II suffit de disposer espèces et relevés selon leur répartition calculée au long de cet axe, pour 
obtenir d'emblée un premier tableau déjà bien élaboré. Ce n'est en général pas le tableau définitif ; des retouches sont nécessaires pour l'affiner et mieux faire ressortir les coupures qu'il apparait souhaitable de matérialiser. Ces retouches peuvent avoir aussi pour but de mettre en évidence des faciès particuliers dont l'originalité est en fait sous la dépendance d'autres facteurs.

Si un ou plusieurs facteurs ont également une place importante, il peut être utile de faire des tableaux partiels, qui seront alors diagonalisés en s'aidant des résultats de l'analyse sur les axes correspondants.

Si les relevés sont trop nombreux pour figurer tous dans un tableau de taille « raisonnable », un échantillon plus restreint peut-être retenu, dont le choix sera, là aussi, largement facilité par les résultats de l'analyse factorielle.

\section{4. - Résultats}

\section{1. - Interprétation des axes factoriels}

Deux premiers traitements ont d'abord eu lieu, l'un en tenant compte du coefficient d'abondance-dominance des espèces, l'autre en présence-absence. Ils confirment les conclusions obtenues sur d'autres dossiers : les résultats sont très semblables, mais il apparait, au moins en forêt, que la prise en compte des coefficients apporte pourtant une légère information supplémentaire, car l'interprétation des axes d'ordre assez élevé (axe 3, 4 ou 5) s'en trouve en général facilitée. Nous reviendrons sur ce point dans une prochaine note de synthèse. Les résultats présentés ici sont donc ceux obtenus en utilisant l'abondance-dominance.

Les pourcentages de variation absorbés par les six axes extraits sont les suivants : $4,42,3,39,3,09,2,37,2,14,1,86$. Leur interprétation est présentée séparément, mais, en règle générale, l'observation de plans factoriels est en fait encore plus fructueuse pour comprendre l'organisation des faits observés. En particulier ici, le plan factoriel des axes 1 et 2 est apparu riche d'enseignement pour la suite de l'interprétation.

\subsection{Interprétation de l'axe 1.}

Sur la figure 1 ont été portées les espèces participant de façon significative à l'élaboration de l'axe 1 et/ou de l'axe 2 ; le critère de ce choix est la valeur du cosinus évoqué au $\$ 3.31$, que l'on a fixée à un minimum de 0,22 .

L'interprétation de l'axe 1 est immédiate. Vers le bas de la figure apparaissent des espèces telles que Quercus lanuginosa (177 ef 178), Sesleria coerulea (211), Polygonatum odoratum (163), Lithospermum purpureo-coeruleum (124) ... qui, dans le Nord de la France, sont des calcicoles xérophiles ; puis des calcicoles plus banales, Cornus mas (58), Cornus sanguinea (59), Viburnum lantana (241) ; puis des neutro-mésophiles, Milium effusum (134), Deschampsia coespitosa (70), Luzula pilosa (127)... ; enfin des mésoacidiphiles à acidiphiles, Atrichum undulatum (32), Lonicera periclymenum (251), Luzula albida (126)... II y correspond un gradient de $\mathrm{pH}$ très net (lui-même fortement corrélé aux possibilités d'alimentation en eau), qui apparaît donc être le facteur de variation stationnelle le plus important. 


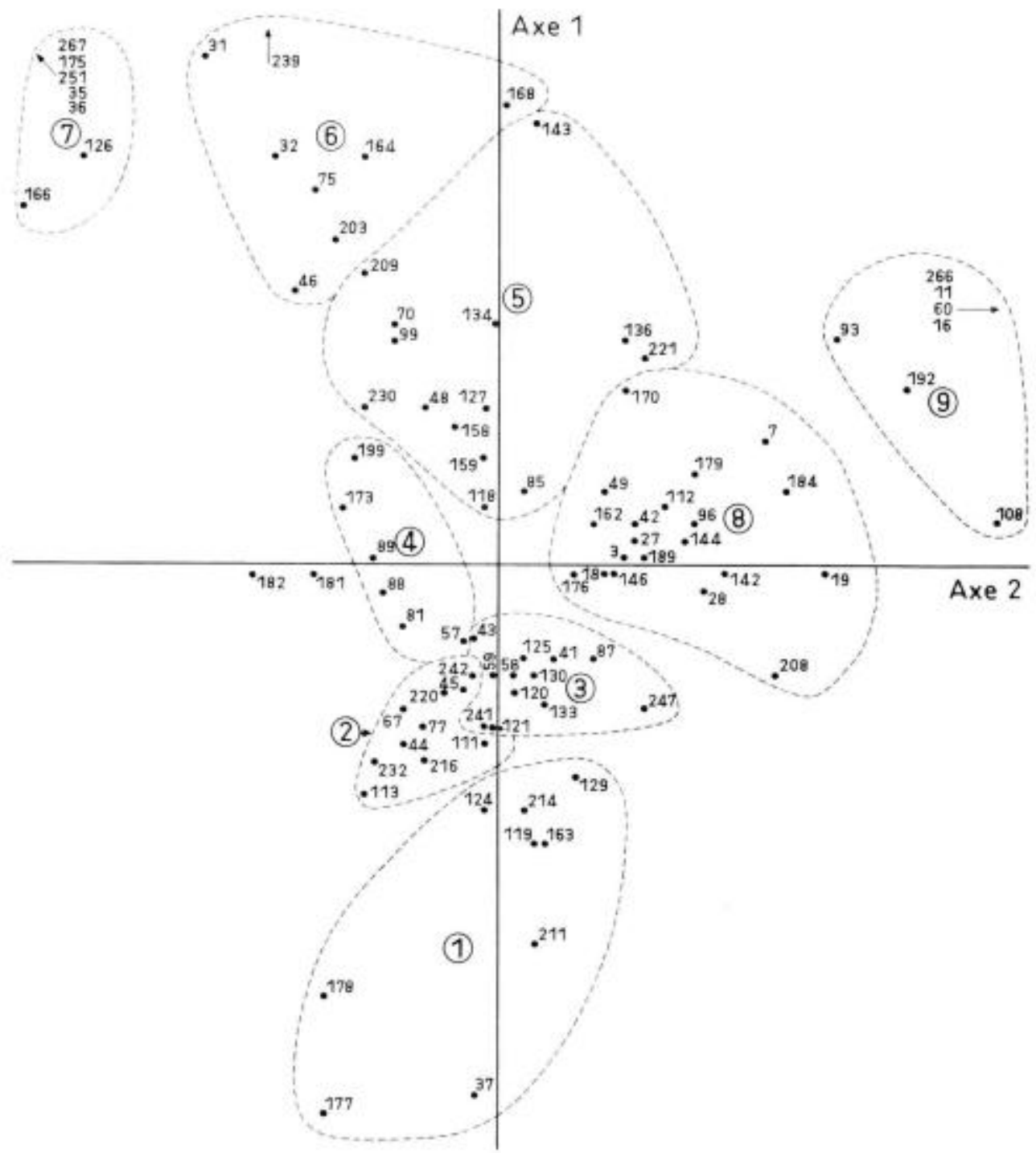

FIG. 1. - Distribution des espèces participant de façon significative à l'élaboration des axes factoriels 1 et / ou 2. Les poinfillés rassemblent les espèces appartenant do un méme groupe écologique (voir $\$ 4.2$ ).

Distribution of species which significantly participate in computation of factor axis 1 and/or 2. Stipplings gather species which belong to the same * ecological group w (see \$ 4.2).

\subsection{Interprétation de l'axe 2.}

L'axe 2 n'oppose pas nettement un groupe d'espèces à une extrémité à un autre à l'autre extrémité. On remarque plutôt, vers la droite, un ensemble d'espèces non spécifiquement calcicoles, mais réputées exigeantes sur le plan de la nutrition minérale et hydrique, caractérisant bien le mull eutrophe; ainsi : Arum maculatum (27), Paris 
quadrifolia (144), Ornithogalum pyrenaĩcum (142) ... A l'extrémité de l'axe apparaissent même des espèces très exigeantes, ayant de plus un caractère nitrophile plus ou moins marqué : Glechoma hederaceum (108), Urtica dioïca (266), Adoxa moschatellina (11)...

II s'agit donc d'un axe « nutrition ».

Le graphique homologue 1-2 pour les relevés (non présenté ici) montre que le nuage des sept cent soixante-douze relevés a, comme celui des espèces, une forme nettement triangulaire, avec une densité de points assez similaire dans les trois directions. Comme par ailleurs les pourcentages de variation absorbés par les deux premiers axes factoriels ne sont pas très différents ( 4,42 et 3,39 p. 100), cela prouve que le facteur du milieu responsable de la matérialisation de ce deuxième axe est loin d'être négligeable vis-d̀-vis du premier. On peut donc d'emblée s'attendre à des difficultés pour l'élaboration du tableau phytosociologique de synthèse, dont la « diagonalisation » doit s'appuyer sur un facteur primordial. Cette question sera reprise dans le $\$ 4.2$.

\subsection{Interprétation de l'axe 3.}

L'interprétation de l'axe 3 est plus délicate, mais fertile encore en enseignements. Sur la figure 2, seules les espèces significatives pour l'axe 3 ont été portées.

Sur la gauche, on relève un certain nombre d'espèces ligneuses : Fagus silvatica $(88,89)$. Acer pseudoplatanus (7 et 8 ), A. platanoides (5 et 6), Fraxinus excelsior (97). Ulmus scabra (235 et 236), et des espèces compagnes: Asperula odorata (29), Elymus europaeus (73), Euphorbia amygdaloides (81)...

Sur la droite, Quercus sessiliflora (181 et 182), Quercus pedunculata (179 et 180), Carpinus befulus (50), Tilia cordała (229 ef 230), accompagnées de Solidago virga aurea (214), Viola mirabilis (247) ... et de nombreuses mousses : Eurhynchium striatum (85), Thuidium famariscifolium (228), Atrichum undulatum (32)...

En fait, cet axe semble schématiquement opposer la Hêtraie ef la Chênaie au sens large. Mais si le déterminisme de la répartition de ces deux grandes formations était essentiellement d'ordre écologique, il se serait vraisemblablement manifesté sur le $1^{\mathrm{er}}$ ou le $2^{\mathrm{e}}$ axe. L'hypothèse vient alors à l'esprit que le type de traitement et, partant, la composition ef la structure du peuplement arborescent sont, au moins en partie, responsables du phénomène observé. Nous avons analysé de plus près la question en sélectionnant deux ensembles de relevés similaires pour les valeurs prises sur les axes 1 et 2, l'un vers le pôle calcicole de l'espace des facteurs 1-2, l'autre vers le pôle mésoacidiphile. Dans chacun ont été gardés les relevés significatifs pour l'axe 3, par valeur positive ou négative.

Deux tableaux phytosociologiques ont alors été construits, qui seront présentés dans une publication ultérieure. Les conclusions de ces confrontations, différentes pour chacun des milieux, sont schématiquement les suivantes :

\section{Pôle calcicole.}

Pratiquement tous les relevés sont en taillis-sous-futaie. Mais d'un côté, ils sont à base de Chêne, Sessile ou Pédonculé, en position de plateau ou sur les flancs de vallons à orientation générale sud, de l'autre côté ils sont à base de Hêtre et en exposition nord. Quelques relevés de plateau portaient en fait un peuplement en cours de conversion en futaie. 


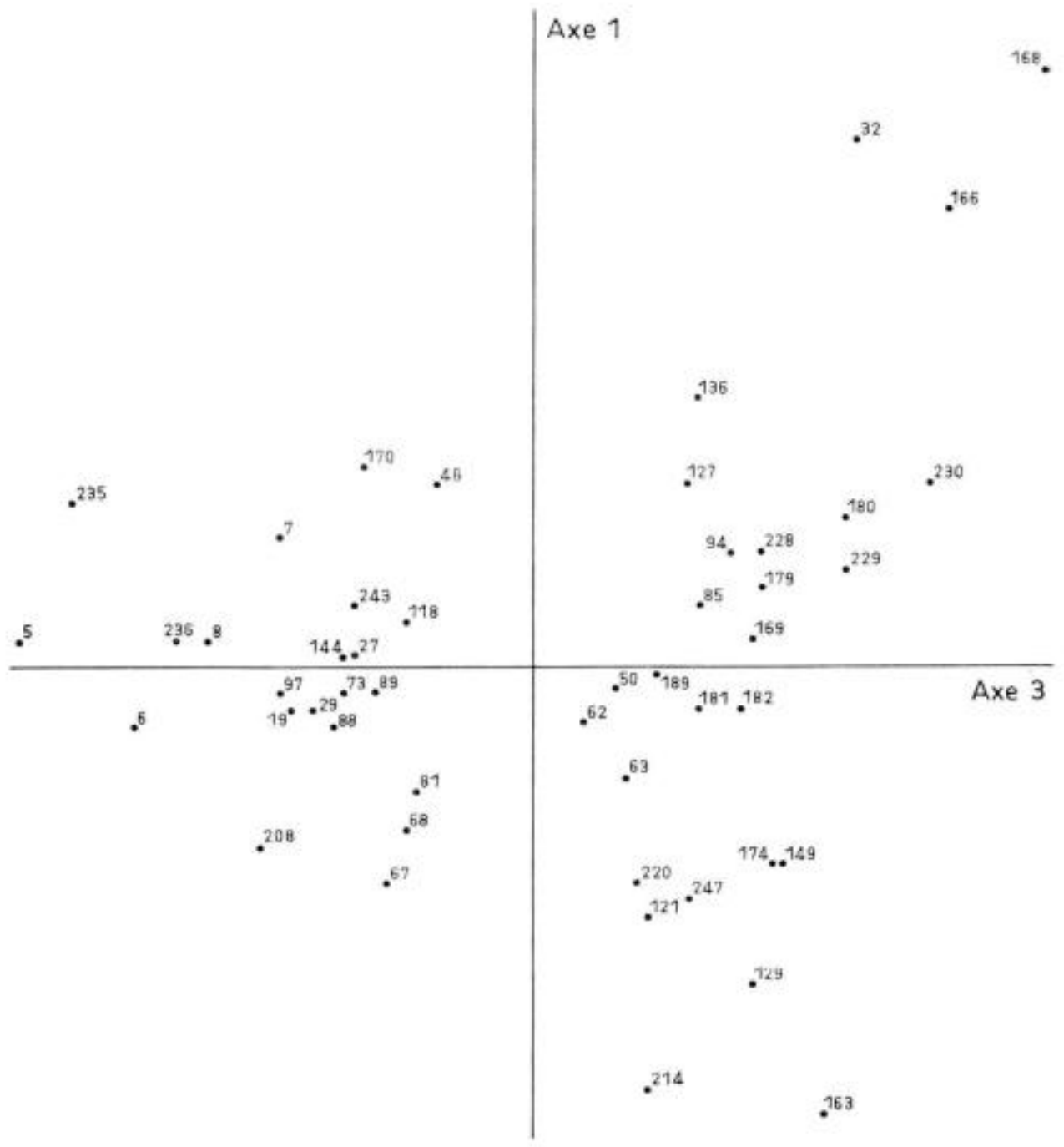

FIG. 2. - Plan factoriel 1-3. Distribution des espèces participant de façon significotive à Pélaboration de l'axe 3.

Plane of factor 1-3. Distribution of species which significantly participate in computation of axis 3.

Pôle méso-acidiphile.

On trouve d'un côté des taillis-sous-futaie à base de Chêne, de l'autre des futaies de Hêtre et quelques peuplements en cours de conversion.

Le point commun des deux types de hêtraie, en dehors de l'essence dominante, nous semble être l'existence, en sous-étage, d'un microclimat thermique et lumineux comparable, et favorable à l'installation ou au maintien du Hêtre et d'un certain nombre d'autres espèces ligneuses et herbacées. Ce microclimat peut être obtenu, soit grâce à la structure de futaie - elle est alors la résultante de l'action humaine - 
soit par le biais d'une exposition Nord marquée, avec pente accusée : dans ce cas, et malgré le traitement imposé, on constate d'ailleurs, à l'expérience, que la physionomie du peuplement et du sous-étage évoque souvent la futaie.

Brièvement, les espèces les plus étroitement inféodées à la hêtraie, dans toute la gamme de stations, sont : Asperula odorata, Euphorbia amygdaloides, Acer platanoïdes, Fraxinus excelsior ; ef de façon plus lâche : Ulmus scabra, Daphne mezereum, D. laureola... Vers le pôle mésoacidiphile, la constance d'Elymus europaeus avec le Hêtre, et lui seul, est tout à fait remarquable. Vers le pôle calcicole, Tilia platyphyllos accompagne de façon très significative le Hêtre sur les versants Nord.

Les espèces spécifiques du traitement en taillis-sous-futaie à base de Chêne sont moins nombreuses et moins strictes. Tilia cordata apparait le plus fidèle dans toutes les situations. Plusieurs mousses peuvent secondairement s'y adjoindre (Eurhynchium striatum, Thuidium famariscifolium, Rhytidiadelphus triqueter...). Vers le pôle calcicole, on peut également citer : Polygonatum odoratum, Solidago virga aurea, Viola mirabilis... Vers le pôle mésoacidiphile, on note Lonicera periclymenum, Populus tremula, Salix caprea, Atrichum undulatum...

Outre l'intérêt pratique et fondamental de la mise en évidence de l'influence du traitement forestier sur une fraction de la flore d'une station donnée, il apparaît donc nécessaire, pour la définition des stations, de tenir compte, en plus des facteurs « $\mathrm{pH}$ » (axe 1) et « nutrition » (axe 2), de l'exposition (axe 3 pour partie).

\subsection{Interprétation des autres axes.}

Les autres axes interprétables n'apportent pas d'information fondamentale pour la définition des stations. Mais il est intéressant d'en faire état pour montrer la puissance d'analyse de la méthode.

La combinaison des axes 4 et 1 individualise à nouveau quelques calcicoles xérophiles d'un côté, mais surtout un groupe d'espèces à caractère nitrophile ou héliophile de l'autre, telles que : Geranium robertianum, Ribes uva crispa, Rubus idaeus, Sambucus racemosa, Eupatorium cannabinum, Epilobium angustifolium, E. montanum, Stachys silvaticus, etc.

En fait, ces espèces se rencontrent, d'une part de façon permanente, au moins la plupart, « en station » dans des situations particulières déjà bien typées par ailleurs (les fonds de vallons), d'autre part de façon circonstancielle et temporaire à l'occasion d'une mise en lumière du sous-bois (coupes d'éclaircie, chablis).

L'axe 5 est encore biologiquement interprétable. II oppose,

- d'un côté des espèces telles que : Epilobium montanum, Hypericum hirsufum, Rubus idaeus, Fragaria vesca, Dactylis glomerata, Taraxacum officinale, Bromus asper, Carex glauca, C. montana, Melampyrum pratense..., qui ont un caractère héliophile plus ou moins marqué,

- de l'autre côté des espèces plus ou moins sciatolérantes telles que Vinca minor, Mercurialis perennis, Eurhynchium striatum, ou liées par d'autres facteurs à des situations ombreuses (Ribes uva-crispa, Glechoma hederaceum, Sambucus racemosa : fonds de vallons), ou créant elles-mêmes une pénombre plus marquée (Acer platanoĩdes, A. pseudoplatanus, Ulmus scabra, Tilia platyphyllos, Fraxinus excelsior : plus abondants dans les futaies denses du massif étudié, ou en exposition ombragée).

L'axe 5 correspond donc, sinon au caractère plus ou moins héliophile des espèces. 
du moins au caractère plus ou moins lumineux des stations où elles se rencontrent le plus fréquemment dans le contexte de l'étude.

Aucune interprétation n'a pu être fournie pour le sixième et dernier axe calculé.

\section{2. - Définition des stations}

L'objectif, exposé au $\$ 3.32$, consiste à élaborer un tableau visualisant les groupes d'espèces et les groupes de relevés que ces derniers permettent de différencier. Les groupes d'espèces sont appelés groupes sociologiques si aucune interprétation écologique n'en est faite, ou groupes écologiques - ou socio-écologiques - au sens de Duvigneaud (1946), si cette interprétation a pu être réalisée. Les groupes de relevés correspondent, soit à des taxons d'ordre divers en phytosociologie classique (alliance, association, sous-association, faciès...), soit à la notion de station si leur déterminisme écologique a bien été établi et qu'une préoccupation d'application pratique directe est sous-jacente (cartographie, aménagement du territoire...).

II serait illusoire, et inutile, de vouloir construire et ordonner un tableau de sept cent soixante-douze relevés; sa présentation matérielle serait de plus malcommode. Le choix de l'échantillon présenté a été guidé essentiellement par la position des relevés dans l'espace des axes factoriels 1 et 2 . Le nuage de points correspondant ayant ( $c$. $\S 4.12$ ) une forme nettement triangulaire, nous avons choisi un certain nombre de relevés en partant du pôle xéro-calcicole (valeurs fortement négatives sur 1 ef moyennes sur 2) pour rejoindre le pôle neutrophile (valeurs moyennes sur 1 et fortement positives sur 2) et terminer par le pôle méso-acidiphile (valeurs fortement positives sur 1 et fortement négatives sur 2 ). De plus, parmi les relevés proches du pôle xéro-calcicole, le plus souvent situés sur pente forte, nous avons pris soin que les expositions sud et nord soient également représentées, afin de tenir compte des informations fournies par l'axe factoriel 3.

Enfin, ayant constaté que dans la pointe neutrophile du triangle se trouvaient, d'une part la majorité des relevés de vallons (mi-pente et bas de pente), mais d'autre part aussi, un nombre important de relevés de plateau, nous avons estimé plus prudent de séparer les deux groupes, bien que la première phase de l'interprétation ne le suggère pas.

De la même façon. un premier arrangement des espèces a été esquissé en tenant compte de leur place dans l'espace des axes 1 et 2 . Après divers remaniements, nous avons estimé que la meilleure façon d'exprimer la réalité de terrain traduite par la forme originale triangulaire du graphique des relevés dans l'espace 1-2, était de construire le tableau en deux parties, l'un, principal, diagonalisé selon l'acidité (axe 1), l'autre, partiel, selon le caractère plus ou moins neutrophile des espèces (axe 2).

Le tableau 1, établi à partir de 61 relevés choisis selon les critères énoncés ci-dessus, synthétise donc notre interprétation phyto-écologique du massif.

Les groupes écologiques en présence.

Nous avons décrit neuf groupes : Les noms qui leur ont été donnés pourront paraître plus ou moins adaptés, mais ils sont pourtant plus parlants que les simples numéros qui seraient suffisants dans l'absolu. En particulier le caractère xérophile des 
premiers groupes est tout à fait relatif : ce sont seulement les plus xérophiles (ou mieux xérotolérants) dans le contexte régional étudié.

\section{Groupe des eu-xérocalcicoles.}

Leur caractère xérotolérant et thermophile est le plus marqué. Certaines espèces sont des intruses en forêt et caractérisent en général des milieux ouverts (Brachypodium pinnatum, Vincetoxicum officinale). La présence de Solidago virga aurea et de Melampyrum pratense peut surprendre ; sauf compensation de facteur non évidente, nous estimons qu'il s'agit de races (écotypes) d'espèces considérées en général comme des acidiphiles.

\section{Groupe des xéro-calcicoles.}

Leur caractère xérophile est moins évident. Ce sont surtout, au moins le premier sous-groupe, des calcicoles strictes. Les deux espèces les plus significatives sont Hepatica triloba et Sorbus aria : pour cette dernière, notons qu'on la retrouve aussi. en dehors du contexte étudié, sur des sols sableux acides dans les Vosges... Mais cela n'enlève rien à son grand intérêt comme espèce indicatrice.

Nous avons rattaché à ce groupe un certain nombre d'espèces semblant avoir, d̀ l'intérieur même du massif, une répartition bimodale. Le cas de Quercus sessiliflora est spécial : le Chêne sessile n'est naturellement pas calcicole au sens strict ; il est beaucoup plus ubiquiste et cède seulement la place au Chêne pédonculé dans certaines stations (voir ci-dessous), ce qui lui confère cette répartition morcelée. Le cas des autres espèces est plus ambigu ; il concorde pourtant avec des observations recueillies par ailleurs, en particulier pour Viburnum opulus et Carex glauca. Ce phénomène, qui méritera une réflexion et une étude plus approfondies pour chaque espèce, enlève cependant de l'intérêt à ces espèces dans le cadre étudié.

\section{Groupe des calcicoles.}

Ce sont les calcicoles « banales », très largement répandues dans le massif, dont seule l'absence peut avoir une signification pour certaines stations. On y trouve la plupart des « morts-bois» des terrains calcaires: Evonymus vulgaris, Ligustrum vulgare, Cornus mas, C. sanguinea, Viburnum lantana, etc...

\section{Groupe des neutrophiles à large amplitude.}

On y trouve toutes les espèces qui, sur plateau calcaire, ne sont pas liées au type de station, sans être pourtant des accidentelles comme les héliophiles (en général non reportées dans le tableau). Rappelons pourtant que la répartition de certaines n'est pas aléatoire, mais liée au type de traitement forestier et à l'essence forestière favorisée (Asperula odorata et Euphorbia amygdaloides en particulier ; cf. $\S 4.13$ ).

\section{Groupe des neutro-mésoacidiphiles.}

L'apparition de ces espèces marque un début de désaturation des humus, sans que l'on puisse encore parler d'acidification. Toutes les espèces du groupe n'ont d'ailleurs pas la même valeur, ainsi que le laisse apparaître la nette diagonalisation à l'intérieur. Les deux espèces les plus significatives sont Deschampsia coespitosa et Milium effusum. 
6. Groupe des mésoacidiphiles.

L'amplitude de ces espèces, à l'intérieur du massif, est beaucoup plus étroite. Certaines ont, de plus, un caractère héliohygrophile (Carex polyrrhiza, $C$. pallescens, Populus tremula, Salix caprea).

\section{Groupe des acidiphiles.}

Nous l'avons distingué du précédent parce que les espèces qu'il contient apparaissent ici de façon marginale, et ont leur optinum dans des milieux nettement acides, non représentés dans le massif (Luzula albida, Polytrichum formosum, Pferidium aquilinum en particulier). Cerłaines sont également des héliohygrophiles plus ou moins marquées (Lonicera periclymenum, Molinia coerulea).

8. Groupe des neutrophiles.

Là aussi nous n'avons pas voulu subdiviser davantage ce groupe, bien que l'amplitude de répartition des espèces soit assez variable : simple tendance pour les premières espèces (Carex silvatica, Lamium galeobdolon...), caractère très marqué pour les dernières (Paris quadrifolia, Ranunculus auricomus, Primula elatior.

9. Groupe des neutro-hygro-nitrophiles.

Ces espèces ont une haute valeur indicatrice, mais caractérisent des milieux très peu répandus.

En plus des espèces apparaissant dans ce tableau partiel (soixante-et-un relevés sur sept cent soixante-douze) quelques-unes, assez rares même dans les milieux qu'elle caractérisent bien, méritent d'être mentionnées :

- pouvant être ajoutées aux eu-xérocalcicoles : Berberis vulgaris, Anthericum liliago, Digitalis lutea, Melittis melissophyllum, Rhamnus cathartica, Sorbus domestica, Rubus saxatilis. Quelques «pseudo-acidiphiles 》 peuvent leur être ajoutées qu'il n'est pas rare, dans d'autres massifs aussi, de rencontrer sur sols calcaires superficiels (écotypes, compensations de facteurs ?) : Dicranum scoparium, Rhamnus frangula, Teucrium scorodonia, Maianthemum bifolium ;

- pouvant être ajoutées aux neutrophiles : Corydallis solida, Colchicum autumnale, Gagea lutea ; et aux neutro-hygro-nitrophiles: Ribes rubrum, Urfica dioïca, Sambucus racemosa ;

- parmi les neutro-mésoacidiphiles : Circaea lufetiona ; et les acidiphiles: Carex pilulifera :

- quelques espèces enfin de climat montagnard, dans certains fonds de vallons froids ou en exposition Nord: Actaea spicata, Festuca silvatica, Centaurea montana, Dentaria pinnata, Lathraea squamaria.

Les stations définies.

L'examen du tableau 1 montre que dix principaux types de station ont pu être définis à l'aide de ces neuf groupes écologiques, appuyés par d'autres données relatives au milieu (topographie et sols). En particulier la correspondance avec les types de sols a pu être établie à partir de la carte au 1/25000 dressée par Gury (1972). Chaque station est caractérisée, essentiellement, par une combinaison originale de groupes écologiques. 
A) Hêtraie-chênaie pubescente de haut de versants Sud.

Elle est caractérisée par les groupes 1 à 4 . Seules quelques rares neutrophiles (groupe 8) peuvent parfois y prendre pied. C'est le groupe 1 (eu-xérocalcicoles) qui lui confère essentiellement son originalité, et en particulier Quercus lanuginosa et Polygonatum odoratum. Ce fut d'ailleurs une surprise de constater que le Chêne pubescent était beaucoup plus fréquent à l'intérieur du massif qu'on ne le supposait auparavant. Son identification n'est d'ailleurs pas toujours très aisée (faute d'accès facile aux feuilles) : il semble de plus que des formes de passage avec les deux autres espèces soient courantes.

Les sols sont pratiquement exclusivement des rendzines brunifiées, très rarement des rendzines noires colluviales, toujours carbonatées dès la surface, sur calcaire dur démantelé ou, plus souvent, colluvium très grossier ; la faiblesse des réserves en eau est aggravée par l'effet combiné de la pente et de l'exposition. L'humus est un mull calcique.

B) Hêtraie-chênaie sessile xérocalcicole de versants Sud.

Par rapport à la station précédente, le groupe 1, encore présent, est nettement réduit. Le Chêne pubescent est encore fréquent. Par ailleurs le groupe 8 (neutrophiles) est déjà bien représenté, au moins par la première partie du groupe (jusqu'à Geum urbanum).

Les sols sont, sur le plan génétique, identiques aux précédents, mais leur régime hydrique doit être un peu moins défavorable.

C) Hêtraie à tilleul calcicole de versants Nord.

Le groupe 1 disparait. Les mêmes autres groupes sont représentés, mais avec quelques nuances importantes. Parmi les xérocalcicoles (gr. 2), quelques espèces ont une fréquence élevée tout à fait spécifique : en particulier Tilia platyphyllos, mais aussi, de façon plus inattendue, Daphne laureola et Clematis vifalba. Par ailleurs, à l'inverse, un assez grand nombre d'espèces ont ici une fréquence d'apparition nettement plus faible que dons les autres stations à caractère calcicole accusé ; mis à part Ribes alpinum, Cornus sanguinea ef Acer campestre, il s'agit surtout de la plupart des « morts bois» calcicoles (Evonymus vulgaris, Viburnum lantano, Ligustrum vulgare, Crataegus sp., Pirus sp., Lonicera xylosteum, Sorbus torminalis...). Mais on relève également Carpinus betulus, Carex monfana, Campanula trachelium, Brachypodium silvaticum. Fragaria vesca, Potentilla fragariastrum.

Ces observations, comme toutes celles relatives à la description des stations, ne s'appuient pas seulement sur le tableau simplifié 1 , mais sur la distribution des espèces dans l'ensemble des sept cents soixante-douze relevés. Elles sont en accord avec les conclusions concernant l'influence de l'exposition exposées plus haut (interprétation de l'axe 3 ; of $\$ 4.13$ ). Malgré le traitement en taillis-sous-futaie, cette station donne souvent une « impression » de futaie, due justement à une densité moindre des arbustes composant le taillis, dont les exigences photiques doivent être insuffisamment satisfaites (exposition).

Les sols sont souvent encore des rendzines brunifiées, mais on relève aussi une proportion notable ( 30 p. 100$)$ de renzdines noires colluviales. Le régime hydrique doit être plus favorable. 
D) Hêtraie-chênaie sessile calcicole de plateau.

Le groupe 1 est très faiblement représenté. Par contre, on note l'apparition d'une première moitié de neutro-mésoacidiphiles (gr. 5), en particulier de Poa chaixì et de quelques mousses (Eurhynchium strialum, Thuidium famariscifolium, Rhytidiadelphus triqueter). Selon les vicissitudes du traitement passé, Fagus silvatica ou Quercus sessiliflora peuvent dominer.

Les sols sont, soit des rendzines brunifiées de plateau (sur calcaire en place). soit des « associations de sols» rendzines brunifiées-sols bruns calciques.

On note également une faible proportion de sols bruns calciques peu profonds ; l'humus passe alors au mull eutrophe. Les réserves en eau du sol sont toujours médiocres.

E) Hêtraie-chênaie neutrocalcicole.

Par rapport à la station précédente, le groupe 1 disparait définitivement, tandis que se renforce très fortement le groupe 8 des neutrophiles. Nous n'avons pas jugé bon de conserver une coupure primitive en deux faciès plus ou moins neutrophiles, qui étaient mal discriminés par ailleurs (sols, productivité, qualité des produits). On arrive là à la limite d'extension de deux espèces à haute valeur indicatrice (stations défavorables) : Sorbus aria et Hepatica friloba. Fagus silvatica ou Quercus sp. (sessiliflora et pedunculata) peuvent y dominer selon les cas. Acer platanoïdes, A. pseudoplatanus (arbustifs) et Carpinus betulus (arborescent) deviennent courants.

Les sols sont le plus souvent des associations rendzines brunifiées-sols bruns calciques, plus rarement des rendzines brunifiées ou des sols bruns calciques typiques. Le régime hydrique est un peu plus favorable.

F) Hêtraie-chênaie pédonculée de vallons.

Le groupe 2 (xéro-calcicoles) se raréfie fortement, tandis que se renforcent les neutro-mésoacidiphiles (gr. 5). Le groupe 8 (neutrophiles) est à son optimum. On peut distinguer la station-type $F 1$ et une variante $F 2$ correspondant à certains fonds de vallons riches, très bien alimentés en eau, plus spécialement caractérisée par les espèces du groupe 9 (dont beaucoup sont d'ailleurs des vernales fugaces : Ficaria verna, Allium ursinum, Adoxa moschatellina, Corydallis cava....).

Quercus sessilifiora cède totalement la place à $Q$. pedundulata. Acer platanoïdes, A. pseudoplatanus, Carpinus befulus, Ulmus scabra sont fréquents.

On a d̀ nouveau affaire essentiellement à des rendzines brunifiées colluviales (à mull calcique), plus rarement (certains fonds de vallons) des sols bruns calcaires (à mull eutrophe). La position topographique fait que, malgré le type génétique, ces sols ont un régime hydrique beaucoup plus favorable (micro-climat différent, apports d'eau latéraux).

G) Hêtraie-chênaie neutrophile de plateau.

Ainsi que le laissaient présager les résultats de l'analyse des correspondances, la composition floristique de cette station est très proche de la précédente, malgré une situation topographique très différente. Tout au plus peut-on constater que le groupe des neutrophiles ( $\mathrm{gr}$. 8) est légèrement moins affirmé. En revenant aux espèces individuelles, rappelons que Sorbus aria et Hepatica triloba sont désormais absentes, alors 
que deux autres espèces à haute valeur discriminante deviennent omniprésentes : Deschampsia coespitosa et Milium effusum. Le Chêne, quand il est présent, est surtout Quercus pedunculata en taillis-sous-futaie, $Q$. pedunculata ou $Q$. sessiliflora en futaie,

Sur le plan pédologique par contre, cette station diffère nettement de la précédente : on a le plus souvent affaire à des sols bruns calciques assez épais, parfois des sols bruns eutrophes ou bruns légèrement lessivés. Les profils sont décarbonatés sur 30 à $40 \mathrm{~cm}$; I'humus est un mull eutrophe à taux de saturation encore élevé $(90$ p. 100 environ). Les réserves en eau utile, parallèlement à l'épaisseur du sol, sont nettement plus élevées que dans les stations précédentes (le régime hydrique par contre doit être assez semblable à celui de la station précédente F).

\section{H) Hêtraie mésoneutrophile.}

Hormis quelques-unes des espèces à répartition supposée bimodale, le groupe 2 des xérocalcicoles disparaît définitivement. Les neutrophiles ( $\mathrm{gr}$. 8) perdent considérablement de leur importance.

Les sols sont voisins génétiquement des précédents, surtout caractérisés par un taux de saturation moins élevé (environ 75 p. 100) de l'humus, qui est encore un mull eutrophe. L'argile de décarbonatation (terra fusca), qui est aussi à l'origine de tous les sols précédents, est ici souvent polluée d'un peu de limons d'origine éolienne ou fluviale.

Remarque. - Dans les forêts communales situées à l'ouest du massif, l'ancienneté du traitement en taillis-sous-futaie a souvent provoqué une substitution d'essence principale, et c'est Quercus pedunculato, en général, qui a remplacé le Hêtre. Mis à part les phénomènes évoqués au $\$ 4.13$, il n'y a pas d'incidence sur la composition des groupes écologiques indicateurs de station potentielle.

I) Hêtraie-chênaie mésoacidiphile.

Cette station, bien typée, est caractérisée par la raréfaction très prononcée des calcicoles (celle des morts-bois est en particulier physionomiquement très caractéristique) et l'installation très marquée des mésoacidiphiles ( $\mathrm{gr}$. 6) et des acidiphiles (gr. 7). Atrichum undulatum et Luzula albida sont les deux espèces les plus caractéristiques.

La couverture limoneuse et/ou alluviale par dessus la terra fusca est ici constante et plus épaisse, et favorise le développement des phénomènes de lessivage. Le sol est le plus souvent du type brun lessivé, ou parfois, lorsque la décarbonatation est plus profonde, du type lessivé. L'humus est un mull mésotrophe, parfois un mull acide, à taux de saturation proche de 50 p. 100.

La remarque faite pour la station précédente $H$ peut également être formulée ici.

I') Chênaie sessile. Hêtraie mésoacidiphile.

Alors que dans les deux situations précédentes, nous avons évoqué la possibilité d'une simple substitution d'essence, nous considérons la station I' comme une forme de dégradation véritable de la station I (ou même de la station $\mathrm{H}$ ) consécutive au traitement forestier passé. Elle est en effet exclusivement située dans un petit massif 
annexe à l'ouest du massif principal, composé de petites forêts communales oủ le taillis-sous-futaie, toujours en vigueur, a été extrêmement « dur », sur des parcelles minuscules. Notre expérience acquise par ailleurs (Becker, 1971) nous incite d̀ conclure que, sur ces matériaux épais et peu filtrants, ce traitement a pu favoriser le développement du lessivage et, à la longue, l'apparition de phénomènes d'hydromorphie ; parallèlement Fagus silvatica, climaciquement à sa place, a été presque totalement remplacé par Quercus sessiliflora (au lieu de $Q$. pedunculata comme précédemment), plus tolérant à l'hydromorphie.

On constate la disparition presque complète des neutrophiles ( $\mathrm{gr} .8$ ) et, plus curieusement, la raréfaction des mésoacidiphiles (gr. 6), excepté Atrichum undulatum. On peut d'ailleurs distinguer deux variantes I'1 ef l'2, la seconde correspondant à une hydromorphie plus accusée, caractérisée par l'extension de Lonicera periclymenum et l'apparition de Molinia coerulea.

Les sols sont du type brun lessivé marmorisé, lessivé marmorisé ou même lessivé à pseudogley. L'humus passe au mull acide, voire au moder.

L'ensemble de l'articulation des groupes écologiques dans les diverses stations est résumée dans le tableau 2.

Remarques générales concernant les stations :

- Bien que telle ne soit pas notre préoccupation majeure dans le cadre de cette étude, il peut être utile de tenter de voir comment les stations ainsi définies prennent leur place dans la systématique phytosociologie classique.

Celle-ci est d'ailleurs encore inachevée et mouvante, et les correspondances proposées n'ont que valeur d'indication.

La plupart des stations appartiennent ou dérivent de l'alliance du Fagion silvaticae, représentée ici par trois sous-alliances :

- le Cephalanthero-Fagenion : on est en présence de l'association du CariciFagefum (stations A, B et D) ou de la sous-association tilietosum (station de versants Nord C). Mais le traitement en taillis-sous-futaie peut localement la faire évoluer vers une chênaie-charmaie de substitution, que I'on pourrait rattacher au Querceto-Carpinetum primuletosum veris (stations B ef D), appartenant à l'alliance du Carpinion. De plus les stations A et B ont souvent aussi de fortes affinités avec l'alliance du Quercion pubescenti-petraeae, association du Querceto-Lithospermefum.

- le Daphno-Fagenion : deux associations semblent représentées : d'une part I'Elymo-Fagefum (station E), avec la forme de substitution à rattacher au Corpinion, le Querceto-Carpinetum medioeuropaeum primuletosum ; d'autre part le Lathyro verni fagetum (stations F1 et G, avec la forme de substifution du Carpinion, le QuercetoCarpinetum alliefosum) et sa sous-association alliefosum (station F2) ;

- I'Eu-Fagenion : elle serait représentée par une association de base, le MelicoFagefum, à laquelle le traitement forestier a pu localement substituer un QuercetoCarpinetum caricetosum glaucae du Carpinion (station $\mathrm{H}$ ) ef une sous-association, le Melico-Fagefum luzuletosum (station 1), pouvant également évoluer vers le Carpinion sous la forme d'un Querceto-Carpinefum medioeuropaeum luzulefosum (station I').

- En forêt domaniale, on constate que c'est dans les stations les plus favorables ( $F, G, H$ ef I) que la conversion en futaie a été engagée en premier, et en général bien réussie. Le processus apparaît aujourd'hui beaucoup plus difficile et aléatoire à 
TABLEAU 2

Caractérisation schématique des stations par les divers groupes écologiques

(— gr. très représenté ; -... gr. moyennement représenté ; $\cdots \cdot$ gr. peu représenté)

Simplified definition of the sites by the different ecological groups (__ very present group ; .... middlingly present group ; .... little present group)

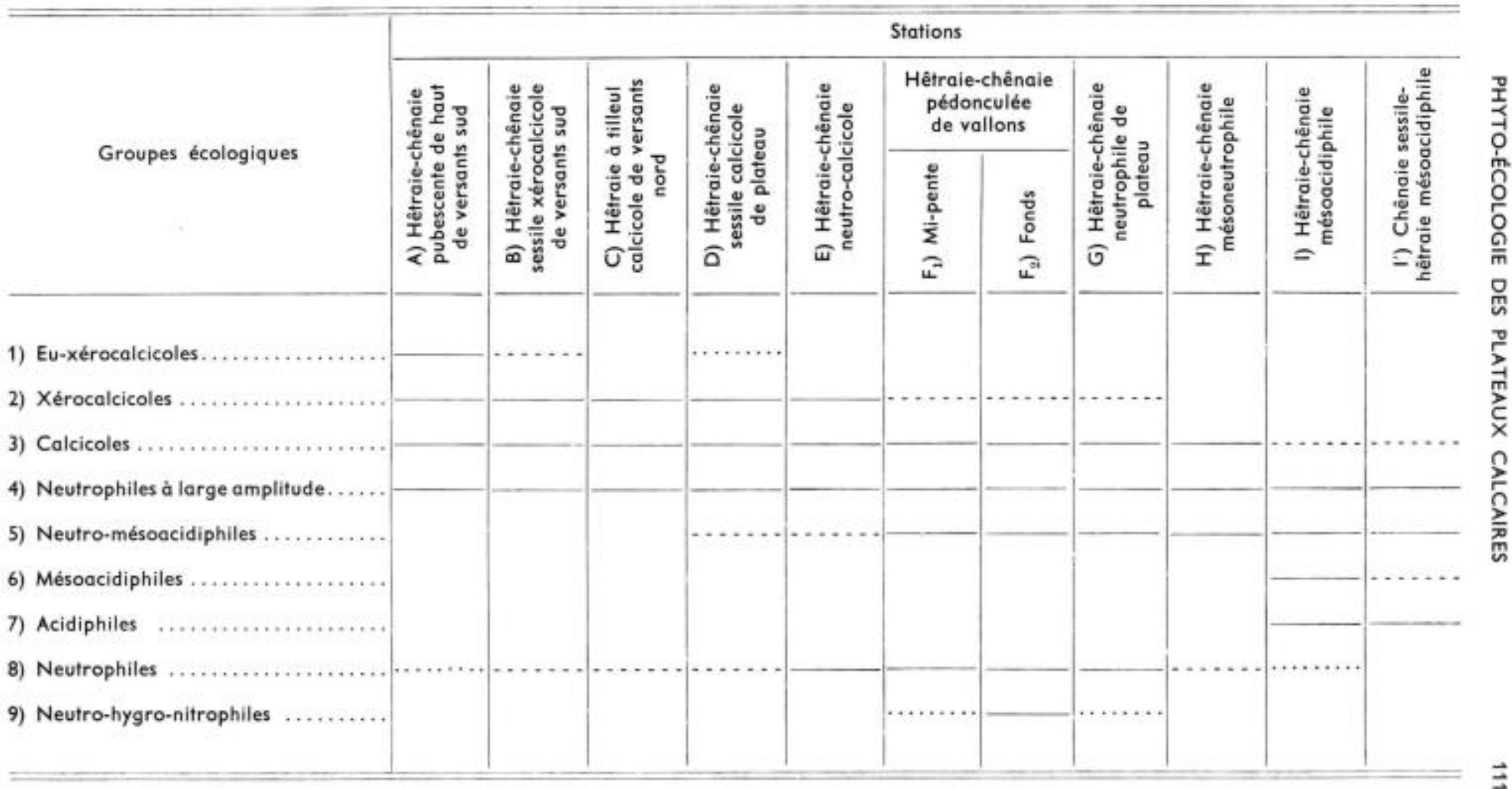


mettre en place dans les stations plus calcicoles, pour des raisons diverses : fructification du Hêtre, survie des semis (Becker, Dubois, Le Tacon, 1977).

- Une bonne corrélation existe entre groupements végétaux ef types de sols. II n'y a cependant pas identité de classification lorsque l'on s'intéresse plus particulièrement à la notion de station, plus synthétique et immédiatement utilisable pour une application pratique. Le type de sol « rendzine brunifiée» en particulier se rencontre dans plusieurs types de stations qu'il est indispensable de distinguer et que la végétation discrimine parfaitement. Inversement, les stations $F 1$ et $G$, très semblables quant aux groupes écologiques en présence, sont caractérisées par des types de sols, des humus et des situations topographiques bien différents. Nous verrons plus loin que des compensations de facteurs doivent intervenir, que la végétation spontanée intégrerait, car la productivité et la qualité des peuplements est très voisine ; nous estimons cependant préférable de les séparer, quitte à laisser le soin au gestionnaire d'opérer des regroupements ultérieurs. Il apparaît donc une fois de plus indispensable de ne jamais dissocier végétation et sols dans la définition ef l'étude des stations.

\section{3. - Carte schématique des stations}

Nous avons vu que l'échantillon de relevés présenté dans le tableau 1 avait été choisi en fonction des coordonnées de ces derniers sur les axes factoriels 1, 2 et accessoirement 3. Mais, de la même façon, en partageant le plan des axes 1 et 2 en secteurs correspondant aux diverses stations retenues (en faisant de plus intervenir l'axe 3 pour la station $C$ et en tenant compte de la position topographique - plateau ou vallon - pour discriminer $F$ et $G$ ) il est aisé de placer chacun des 772 relevés inventoriés dans une station définie.

La carte ainsi obtenue (voir annexe), compte tenu du maillage relativement lâche adopté pour les points-relevés, ne peut être valablement présentée qu'au 1/50 000. Cette échelle est naturellement trop petife pour une utilisation directe dans le détail par le gestionnaire ; elle pourra cependant lui fournir, par «séries 》 (unités de gestion), des indications générales sur la qualité moyenne des stations ef leur répartition par grandes unités.

Toutefois, il lui sera relativement facile, à l'aide des résultats de l'étude, de dresser « au coup par coup», à l'occasion de révisions d'aménagement par exemple, de véritables cartes des stations (1/10 000 en général). Des indications d'ordre pratique (groupes écologiques simplifiés en particulier) sont proposées dans un article séparé (Becker, 1978).

\section{Distribution actuelle des principales essences.}

La carte présentée comporte un petit carton annexe indiquant la répartition actuelle du Hêtre, du Chêne sessile et du Chêne pédonculé. Le Chêne pubescent, trop localisé, n'a pu être indiqué, du fait de l'échelle trop petite, et a été assimilé au Chêne sessile. La comparaison avec la carte des stations montre clairement que sur un même type stationnel peuvent se trouver des essences « naturelles » bien différentes. Nous avons vu que le traitement imposé au cours des siècles derniers (taillis-sous-futaie, à coupes de taillis plus ou moins rapprochées, ou futaie) était responsable. 
Le Hêtre, presque partout climaciquement à sa place, est surtout dominant dans les meilleures stations des séries domaniales (oủ la conversion en futaie a été largement entamée depuis un siècle). Dans les stations médiocres, à sols superficiels, le taillis sous-futaie a favorisé le Chêne sessile aux dépens du Hêtre (en particulier à l'est de la carte). Dans les meilleures stations de forêts communales, l'interprétation est un peu plus délicate : lorsque la couverture limoneuse ou alluviale est d'épaisseur moyenne, ou le régime de coupes de taillis un peu moins sévère, c'est le Chêne pédonculé qui remplace le Hêtre (partie ouest du massif principal) ; lorsque les alluvions sont épaisses, propices à l'hydromorphie, et le traitement plus éprouvant, il y a dégradation du milieu (cf. station l') et le Chêne sessile, plus tolérant, devient dominant.

\section{4. - Observations sur la fertilité des stations}

\subsection{Introduction.}

Nous avons construit, pour le Chêne et le Hêtre, des graphiques indiquant la hauteur des arbres mesurés en fonction de leur âge (cf. $\$ 3.22$ ).

Pour le Hêtre, nous nous sommes aidé de la table de production de Schober (1967). utilisable dans le Nord-Est de la France, pour ramener ces hauteurs à un âge de référence (150 ans) et, au moins pour les relevés de futaie, obtenir une idée de la productivité en volume. Pour les relevés de taillis-sous-futaie, en l'absence d'étude précise dans ce domaine, nous avons opéré en supposant la forme des courbes de croissance comparable à celle de futaie, ce qui est vraisemblablement plus ou moins inexact, mais doit toutefois permettre un classement relatif satisfaisant des stations ; il est évident qu'aucun chiffre de productivité ne peut être avancé.

Pour le Chêne (nous n'avons pas distingué Sessile et Pédonculé dans les calculs), nous nous sommes inspiré de la table de Pardé (1962), qui est apparue extrapolable à l'Est de la France ; celle-ci ne comporte qu'une classe unique, et nous avons déduit les autres courbes de croissance par simple homothétie. Les hauteurs mesurées ont ainsi été ramenées à un âge de référence de 200 ans. La remarque concernant le cas du taillis-sous-futaie s'applique également ici.

Les moyennes des hauteurs compensées pour les diverses stations apparaissent dans la figure 3 . Lorsque le nombre de relevés est suffisant (au moins $>10$ ), nous avons porté en pointillé la moyenne des relevés de futaie.

\subsection{Influence du traifement sur la hauteur dominante.}

Hormis le cas du Hêtre dans la station $E$, toutes les différences qui apparaissent sont statistiquement significatives au seuil de 5 p. 100. Même les moyennes calculées sur de trop faibles séries vont dans le même sens. Si nous nous référons à la station $G$ (Hêtraie-chênaie neutrophile de plateau), qui est la mieux équilibrée en nombres de relevés de futaie et de T.S.F., le Hêtre de futaie à $2,3 \mathrm{~m}$ de plus à l'âge adulte, et le Chêne $3,3 \mathrm{~m}$.

Comme on le pressentait, le traifement, et donc la structure du peuplement, ont bien, à station semblable, une influence très marquée sur la hauteur des arbres. 

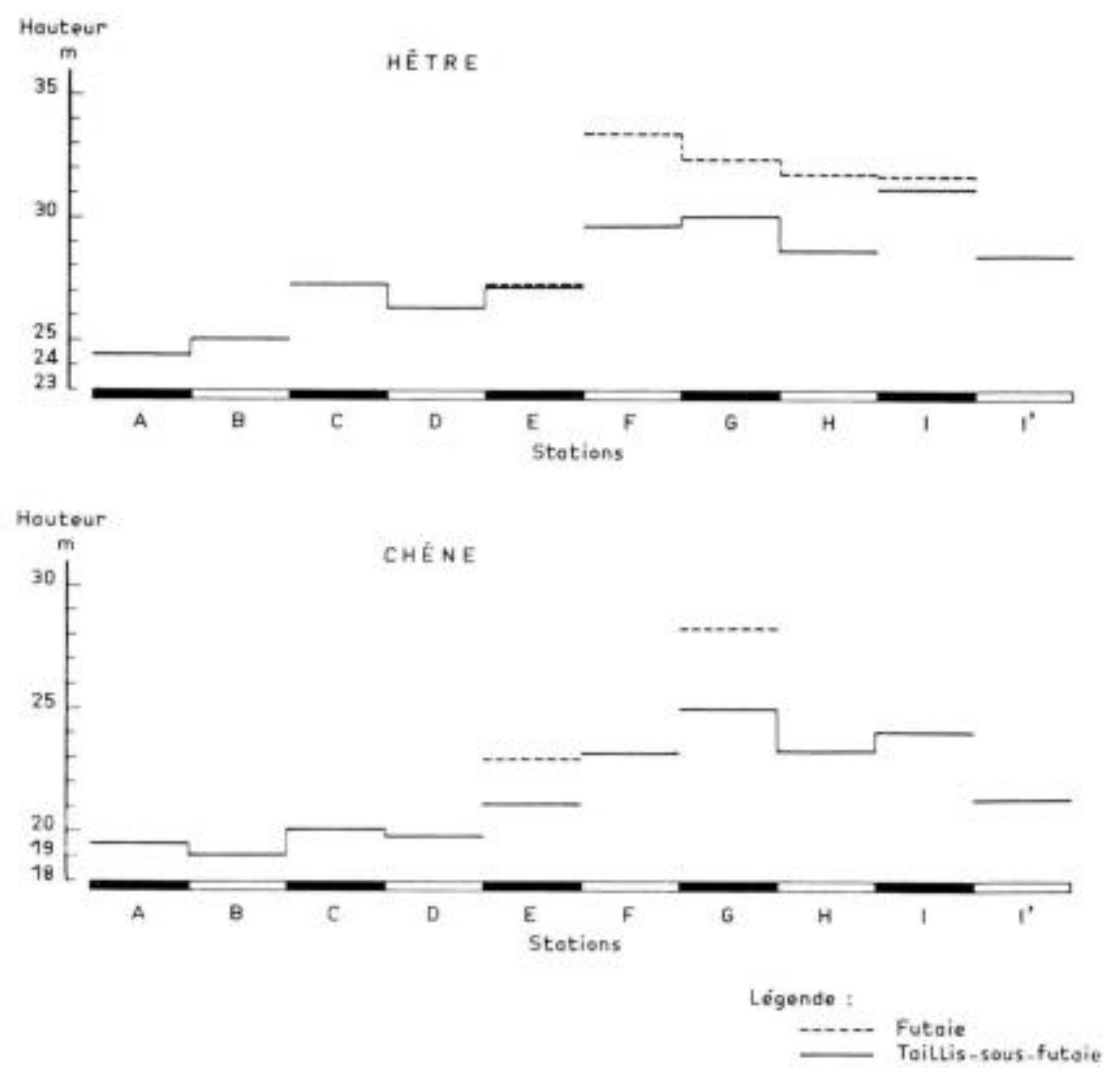

FIG. 3. - Hauteur compensée du Hêtre ef du Chêne dans les stations (deux stations consécutives non différentes statistiquement sont reliées par un trait vertical.

Compensated height of beech and ook in the different sites (two consecutive sites, which are not statistically different, are joined with a vertical line).

4.43. La fertilité des stations. Productivité du Chêne et du Hêtre.

Le classement relatif des stations est très semblable pour le Chêne et pour le Hêtre. Des analyses de variance faites par essence et par traitement forestier ont montré l'influence hautement significative de la station sur les performances atteintes.

Un certain nombre de comparaisons de moyennes ont alors été faifes, stations deux à deux. Beaucoup se sont révélées significativement différentes. En particulier :

Hêtre taillis-sous-futaie : A-C ; B-C ; A-D ; E-F ; E-H ; H-I ; I-I' ...

Hêtre futaie : E-F ; E-G.

Chêne taillis-sous-futaie : A-E ; D-E ; E-F ; F-G ; I-I'.

Chêne futaie : E-G.

Au total, la fertilité apparaît donc bien corrélée au facteur 1 de l'analyse des correspondances, c'est-d̀-dire au caractère plus ou moins calcicole et xérophile des stations. On remarque aussi qu'à la forme de dégradation l' correspond bien une 
baisse de fertilité. Ces résultats concordent avec les observations de Le Tacon et Nys (1970).

II est possible de schématiser en faisant trois grands groupes de stations :

\begin{tabular}{lll} 
& \multicolumn{1}{c}{ Hêtre } & Chêne \\
Stations mauvaises : A, B, D & $4,5 \mathrm{~m}^{3} / \mathrm{ha} / \mathrm{an}$ & \\
Stations médiocres : C, E, I & $5 \mathrm{~m}^{3} / \mathrm{ha} / \mathrm{an}$ & $4 \mathrm{~m}^{3} / \mathrm{ha} / \mathrm{an}$ \\
Stations favorables : F, G, H, I & $6,5 \mathrm{~m}^{3} / \mathrm{ha} / \mathrm{an}$ & $5,1 \mathrm{~m}^{3} / \mathrm{ha} / \mathrm{an}$.
\end{tabular}

(Les chiffres de productivité correspondent à un traitement en futaie, et sont très approximatifs, surtout pour le Chêne ; cf. § 4.41.)

\section{5. - Approche de la qualité technologique des bois}

\subsection{Introduction.}

Les mesures de « couple de torsion» (cf. \$ 3.22) ont pour objet de fournir une indication sur la qualité des bois ; celui-ci est en effet très corrélé à la densité et à la dureté du bois (Polge, Keller, 1970). Par ailleurs, Keller, Le Tacon, Timbal (1976), ont montré, pour le Hêtre traité en futaie, que l'époque de mesure avait une influence sur la valeur du couple de torsion. Nous avons donc également mesuré au laboratoire la densité des échantillons prélevés à la tarière de Pressler, par la méthode de saturation intégrale, pour confirmer ou non le phénomène pour le Chêne, et dans le cas du taillis-sous-futaie.

\subsection{Corrélations entre couple de torsion et densité.}

a) Influence de la date de mesure de $C$.

Cas du Hêtre. - Le coefficient de corrélation global est de 0,38 (significatif au seuil de 1 p. 100) ; l'équation de régression est :

$$
D=421+0,580 \mathrm{C} \quad\left(\mathrm{D} \text { en } \mathrm{g} / \mathrm{dm}^{3} ; C \text { en } \mathrm{cm} \cdot \mathrm{kg}\right) \text {. }
$$

Si l'on calcule des coefficients de corrélation séparément pour des mesures faites au printemps (6 au 31 mai) et en été (21 juillet au 17 août), on obtient respectivement 0,67 et 0,72 . L'époque de mesure a donc bien une influence notable sur les chiffres obtenus pour le couple de torsion (qui correspond en majorité à des relevés de taillissous-futaie).

Cas du Chêne. - Le coefficient de corrélation global est de 0,66 (significatif à moins de 1 p. 100), ef l'équation de régression :

$$
\mathrm{D}=323+0,937 \mathrm{C} \text {. }
$$

Pour les mêmes périodes que ci-dessus, les coefficients passent à 0,77 et 0,80 . Le phénomène « époque de mesure » joue donc également pour le Chêne, mais de façon moins importante semble-t-il.

b) Intérêt relatif du couple de forsion ef de la densité.

Anticipant sur le paragraphe suivant, nous faisons état de neuf comparaisons 
de moyennes entre stations, faites d'une part sur les valeurs de $D$, d'autre part sur celles de C. Les couples de valeurs des écarts réduits ainsi calculés sont les suivants :

\begin{tabular}{|c|c|c|c|c|c|c|c|c|c|}
\hline avec D... & 1,24 & 0,14 & -0.40 & $-0,30$ & 1,32 & 1,72 & 2,37 & 1,98 & 1,61 \\
\hline avec $C \ldots$ & 2,05 & 1,63 & 3,58 & 2,46 & 1,98 & 1.69 & 4,89 & 2,18 & 3,12 \\
\hline
\end{tabular}

De façon assez inattendue, à une exception près, l'écart réduit le plus élevé est obtenu en utilisant le couple de torsion. Celui-ci intègre donc d'autres variables que la seule densité (et de façon non négligeable) et celles-ci sont également liées aux types de stations. La dureté en particulier doit y contribuer notablement. Malgré le «bruit » occasionné par la date de mesure (mais la majorité des relevés sont des relevés d'été), nous avons done préféré approfondir les relations station-qualité des bois en faisant seulement appel aux mesures de couple de torsion.

Cas du Hêtre. - Les moyennes des couples de torsion calculées pour chacune des stations apparaissent dans la figure 4. Nous y avons séparé les relevés de taillissous-futaie et ceux de futaie lorsqu'ils étaient en nombre suffisant (supérieur à 10).

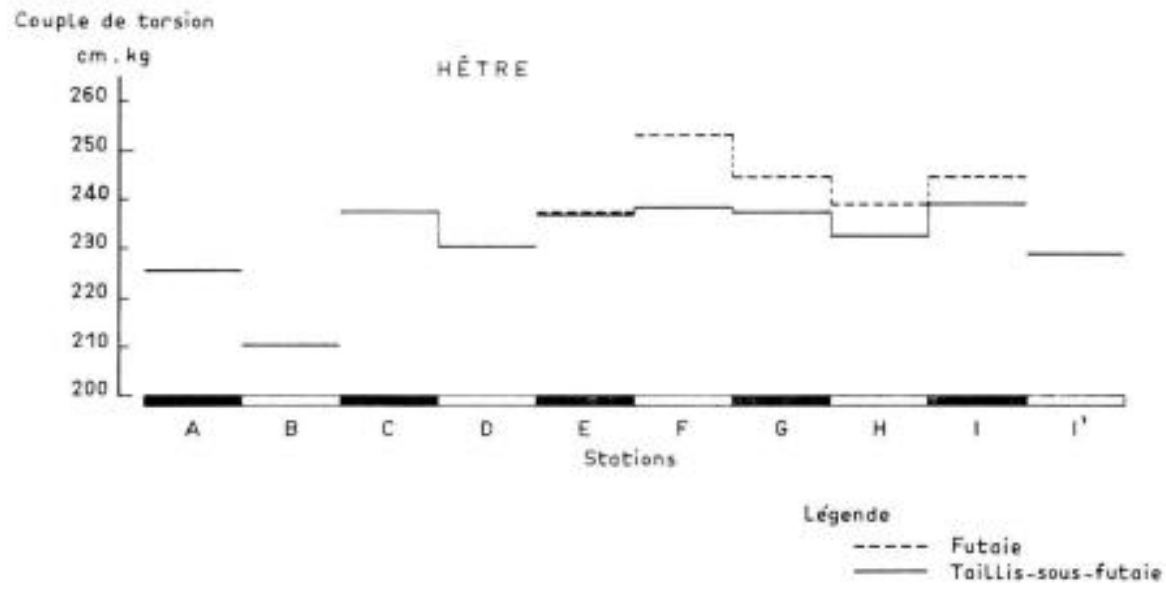

FIG. 4. - Couple de torsion du Hêtre dans les stations

(deux stations consécutives non différentes statistiquement sont reliées par un trait vertical)

Torsion forque of beech in the different sites (two consecutives sites, which are not statistically different, are joined with a vertical line).

Influence du traitement.

Hormis dans la station E (même valeur), le couple de torsion est plus élevé en futaie qu'en taillis-sous-futaie. Mais seule la station $F$ a résisté aux tests statistiques $(\varepsilon=2,73$; significatif à moins de 1 p. 100). Pour la station $G, \varepsilon=1,14$ (significatif d̀ 25 p. 100 seulement).

Ces observations confortent les conclusions de Polge (1973) : les hêtres d̀ cîme large (ici les réserves du taillis-sous-futaie) sont moins durs que ceux à cime étroite (futaie) ; et les hypothèses de Keller ef al. (1976). 
Influence de la station.

Sont entre autres significatives au seuil de 5 p. 100 les différences interstationnelles A-B, A-C, A-E, B-D, D-E, D-F, I-I' en taillis-sous-futaie, E-F, F-H en futaie.

C'est donc dans les stations les plus défavorables (sol superficiel, exposition chaude) que le bois est le plus tendre (le classement relatif des stations A et B est cependant difficile à interpréter). L'exposition peut compenser un sol superficiel (station C). Enfin, la station I' confirme son autonomie par rapport à I sur le plan des caractéristiques du bois également.

\section{Cas đu Chêne. - Les résultats apparaissent dans la figure 5.}

Une première interprétation a été faite sans préoccupation d'espèce. Un certain nombre de différences sont apparues significatives, en particulier A-E, D-E, E-F, F-G, $\mathrm{I}-\mathrm{I}$ ', $\mathrm{H}-\mathrm{I}$ ' (taillis-sous-futaie). Mais une interprétation logique de ces différences, d'ailleurs assez faibles, apparaissait difficile et semblait aller dans le sens d'une opinion tendant à se faire jour, selon laquelle les caractéristiques du bois de Chêne seraient beaucoup moins dépendantes de la station que celles du Hêtre.

L'effet de la structure du peuplement, par contre, apparaissait déjà très marqué : les 4 écarts entre taillis-sous-futaie et futaie apparaissant sur la figure sont hautement significatifs (test de Student dans les cas des échantillons réduits $\mathrm{H}$ et I de futaie).

Nous avons cependant tenté d'approfondir la question en séparant les espèces lorsqu'elles peuvent se rencontrer dans un même type de station avec une forte probabilité. Les interprétations sont alors beaucoup plus aisées.

Elles est surtout évidente pour le Chêne pédonculé, essence à amplitude écologique plus étroite que le Sessile. Ce sont dans les stations les plus riches, à mull eutrophe, celles qui lui conviennent le mieux, que le Pédonculé est le plus dur ; dans les stations où il devient plus marginal, à mull calcique ou à mull acide, le couple de torsion est nettement plus faible (différences statistiquement significatives: E-F, F-G, H-I). Ce résultat est similaire à celui observé pour le Hêtre par nous même (mais en plus net encore) et par Keller et al. (1976).

Quant au Chêne sessile, il vient surtout remplacer le Pédonculé lorsque ses exigences ne sont plus satisfaites ; il est donc pratiquement absent des stations $F$ et $G$ (sauf en futaie). Pour les stations A, B, C, D, E, les différences ne sont pas significatives. Mais, au moins pour $A$ et $B$, une troisième espèce intervient, le Chêne pubescent, qui, de façon hautement significative, est fortement plus dur que le Sessile. Comme des formes de passage existent indéniablement entre les deux espèces, il est permis de penser que les moyennes pour le Sessile stricto sensu sont encore surestimées. Toujours est-il que c'est dans la station I, là oủ il parait le plus indiqué (mais en forte concurrence avec le Hêtre...), que le couple de torsion est significativement le plus élevé. Puis il s'effondre dans la station l', qui confirme une fois de plus son caractère particulier (dégradation ; cf. § 4.2).

Dans les stations où les deux essences, Sessile et Pédonculé, sont susceptibles d'être présentes, c'est le Sessile qui, de façon statistiquement significative, est apparu le plus dur (sauf dans la station « de transition » $\mathrm{H}$ ). 

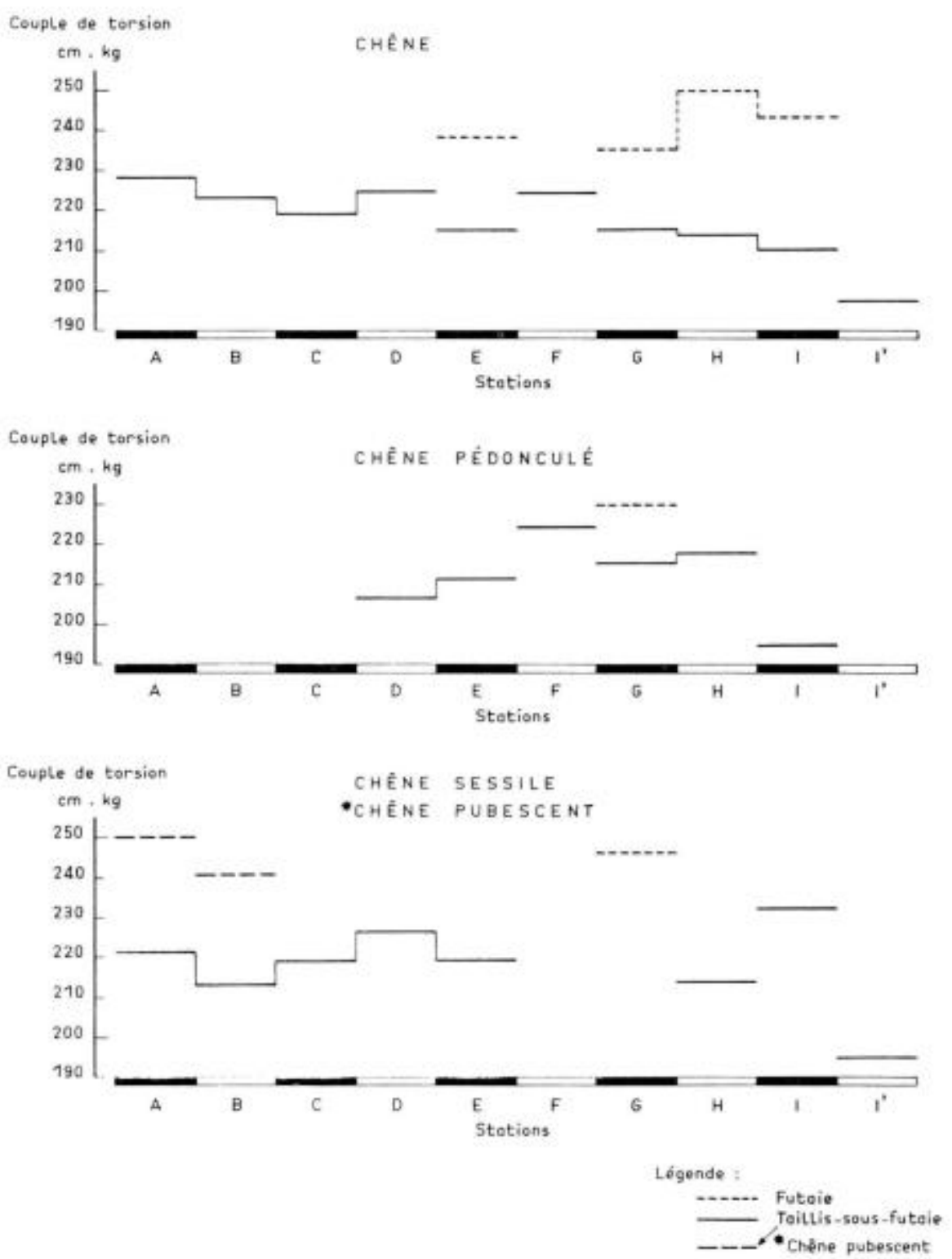

FIG. 5. - Couple de torsion du Chểne dons les stations

(deux stations consécutives non différentes statistiquement sont reliées par un trait vertical).

Torsion torque of oak in the different sites (two consecutive sites, which are not statistically, different, are joined with a vertical line). 


\section{5. - Conclusions}

Sur le plan méthodologique

L'analyse factorielle des correspondances s'est avérée un outil extrêmement commode et puissant pour l'interprétation simultanée d'un grand nombre de données phyto-écologiques, en particulier pour la compréhension des grands facteurs du milieu régissant la distribution des espèces ef pour l'appréciation de l'importance relative de ces derniers dans le cadre d'une étude.

Elle facilite de plus considérablement l'élaboration du tableau phytosociologique des stations, qui demeure de toutes façons indispensable pour la matérialisation définitive des stations. A cette fin, la notion de groupe écologique confirme son efficacité et sa commodité d'utilisation ; chaque station est caractérisée par une certaine combinaison originale de ces groupes, préalablement mis en évidence et interprétés.

Sur le plan des connaissances scientifiques de base

Les apports de l'étude sont d'ordre divers.

Les uns concernent la valeur indicatrice des espèces susceptibles d'être rencontrées dans le contexte plateaux calcaires du Nord-Est de la France, ef leur regroupement en groupes écologiques. Neuf groupes sont ainsi proposés dans le cadre de l'étude, qui répondent à deux gradients écologiques combinés, un gradient de $\mathrm{pH}$. corrélé aux possibilités d'alimentation en eau, et un gradient de qualité de la nutrition minérale et azotée. La constance et la fidélité des espèces individuelles à ces groupes a pu être appréciée. La combinaison des groupes, avec l'appui d'autres données du milieu (sols, topographie) permet de définir 10 types de stations, qui devraient fournir une base solide pour l'établissement d'une typologie régionale.

D'autres, intéressants pour le phytosociologue théoricien comme pour le praticien utilisateur, ont trait à l'incidence du traitement forestier sur la composition floristique. Dans la systématique phytosociologique, qui a sans doute encore mal apprécié l'ampleur possible du phénomène, on peut même passer d'une alliance à l'autre. Mais celui-ci n'affecte seulement qu'une fraction réduite du potentiel floristique (les arbres surtout), et l'utilisation des groupes écologiques permet de retrouver l'identité stationnelle qui se cache derrière deux peuplements dominés par des essences différentes. Dans certains cas pourtant, le milieu lui-même peut évoluer de façon difficilement réversible et l'on a alors affaire à une station différente (l' par exemple).

Enfin, l'étude contribue à la connaissance des facteurs entrant en jeu dans le déterminisme des deux paramètres sylvicoles fondamentaux que sont la fertilité des peuplements et la qualité technologique des produits obtenus. La fertilité apparaît bien corrélée aux types de stations ; mais le type de traitement, futaie ou taillis-sousfutaie, a des répercussions importantes sur les hauteurs dominantes et complique la mise en évidence des liaisons. Quant à la qualité technologique, appréciée par le biais de la mesure du couple de torsion (qui s'est avérée plus discriminante encore que la mesure précise au laboratoire de la densité), son déterminisme apparaît net- 
tement plus complexe : l'espèce, la station et la structure du peuplement jouent simultanément. Pour l'effet-espèce, il apparaît primordial de séparer chênes sessile et pédonculé (et même pubescent dans notre cas). Pour l'effet-station, il se confirme, pour toutes les essences, que la dureté du bois passe par un maximum (et donc la qualité par un minimum) dans les stations où la nutrition est la meilleure. Pour l'effetstructure du peuplement enfin, il se confirme que les houppiers larges du taillis-sousfutaie sont plus favorables que ceux de futaie dans le contexte étudié ; ceci n'est pour autant pas un plaidoyer pour le taillis-sous-futaie, mais un argument pour des éclaircies plus fortes que celles qui y ont été longtemps pratiquées en futaie.

\section{Sur un plan pratique local}

Nous proposons au praticien forestier l'élément écologique pour une meilleure connaissance de ce vaste massif de 12000 ha et la conception d'une gestion aussi raisonnée que possible. La carte ci-jointe fournit des indications sur la répartition générale des stations par grands ensembles. Pour plus de détail, les résultats de l'étude fournissent les éléments nécessaires à une carlographie à plus grande échelle (des indications d'ordre pratique sont proposées dans un article séparé).

Par ailleurs, le massif de Haye, aux portes de Nancy, est un terrain d'expériences et d'observations de choix pour les chercheurs de I'I.N.R.A. Chaque spécialiste peut donc désormais raisonner ses travaux dans un contexte de stations bien définies, ce qui est très souvent primordial.

$$
\text { Reçu pour publication en juillet } 1978 .
$$

\section{Summary}

\section{A phyto-ecological sfudy in the calcareous regions of N.-E. France (Forest of Haye-54) \\ Use of factorial analysis of correspondences for site typology. Relationship with productivity and wood quality of beech and oak}

The forest of Haye $(12000 \mathrm{ha})$ is representative of the extensive forest areas located in the calcareous regions of N.-E. France. Some 800 plant inventories were made, which were completed by dendrometric and technological measurements on the main tree species (beech and oak).

A factorial analysis of correspondences evidenced the decreasing importance (as regards the floristic composition) of some ecological, edaphic or climatic, factors : $\mathrm{pH}$ (which is correlated here with water supply possibilities), mineral nutrition, exposure, nitrogen nutrition... ; but also the significant importance of the silvicultural system (high forest or coppice-with-standards). Moreover this analysis greatly facilitates the elaboration of a synthetic site table (table 1 ). Nine $\alpha$ ecological groups $x$ (species' groups) and ten site units were defined ; each site unit is characterized by an original combination of ecological groups, as well as specific edaphic properties and a specific exposure. These site units have been mapped (see annex). It should be possible to apply this typology very widely to the whole calcareous region under consideration.

Measurements of stand height showed a good correlation between productivity and site units. for both beech and oak (fig. 3), but trees in high forest are taller than in coppice-with-standards.

As for wood quality, the torsion torque method (Polge-Keller, 1970) appeared more discriminative than air-dry density of wood samples (from increment borer). Determinism of the torsion torque proves more complex (fig. 4 and 5) : species, site unit and crop structure interfere. For specieseffect, it is necessary to distinguish befween sessile and pedunculate oaks ; for site-effect, there is a negative correlation befween wood quality and nutrition quality (soil) ; for crop structure effect. large crowns appear more favourable. 


\section{Références bibliographiques}

AUSSENAC G., BECKER M., 1968. Ecologie d'un massif sur sols hydromorphes : la forêt de Charmes (Vosges). Contribution à la mise au point d'une méthode d'étude dynamique du milieu forestier. Ann. Sci. forest., 25, 4, 291-332.

BECKER M., 1969. Le Hêtre (Fogus silvatica L.) et ses problèmes en forêt de Villers-Colterêts (Aisne). Contribution à la mise au point d'une méthode dynamique d'étude écologique du milieu forestier. Ann. Sci. forest., 26, 2, 141-182.

BECKER M., 1971. Etude des relations sol-végétation, en conditions d'hydromorphie, dans une forêt de la plaine lorraine. Thèse Doctorat d'Etat, Université de Nancy 1, 225 p.

BECKER M., 1972. Ecophytosociologie et production ligneuse. Colloque sur les relations entre l'écologie des stations et la production ligneuse. Ann. Sci. forest., 30, 2, 143-182.

BECKER M., DUBOIS F. X., LE TACON F., 1977. Types de station, fructification ef installation des semis de Hêtre sur les plateaux calcaires du Nord-Est (forêt de Haye, 54). Interaction avec le travail du sol ef l'élimination de la végétation concurrente. Rev. forest. franç., 29, 5, 363-374.

BECKER M., 1977. Forêt française : pour une définition et une cartographie des stations. Document Laboratoire de Phylo-écologie du C.N.R.F., 11 p.

BECKER M., 1978. Définition des stations en forêt de Haye (54). Potentialités du Hêtre et du Chêne. Rev, forest. franç., 30, 4.

BENZECRI J. P. ef al., 1973. L'analyse des données : l'analyse factorielle des correspondances. Tome 2, Dunod, Paris, 619 p.

BRIANE J.P., LAZARE J.-J., ROUX G., SASTRE. C., 1974. L'analyse factorielle des correspondances ef l'arbre de longueur minimum ; exemples d'application. Adansonia, sér. 2, 14, 1, 111-137.

DUCHAUFOUR Ph., 1950. Recherches sur l'évolution des sols calcaires en Lorraine. Ann. Ecole Nationale des Eaux ef Forêts, 12, 1, 99-153.

DUMÉ G., 1975. Contribution à l'étude phytosociologique et écologique des forêts à Chêne et à Charme du Bassin parisien au sens large. Thèse de $3^{\circ}$ cycle, Paris-Orsay, 92 p.

DUVIGNEAUD P., 1946. La variabilité des associations végétales. Bull. Soc. roy. Bot, Belg., 78, 107-134.

ESCOUFIER Y., 1972. Analyse des données. Fac. des Sci. univ. Montréal, 124 p.

GURY M., 1972. Carte pédologique du Plafeau de Haye. Notice explicative. C.N.R.S., Centre de Pédologie biologique, Nancy, 46 p. + 1 carte.

JURATIC L., PLAN J., 1976. Etude phyto-écologique en forêt domaniale de Grande-Chartreuse. Mémoire $3^{\circ}$ année E.N.I.T.E.F., 101 p. + annexes.

KELLER R., LE TACONF., TIMBAL J., 1976. La densité du bois de hêtre dans le Nord-Est de la France. Influence des caractéristiques du milieu et du type de sylviculture. Ann. Sci. forest., 33, 1, 1-17.

LACOSTE A., ROUX M., 1971-1972. L'analyse multidimensionnelle en phytosociologie et en écologie. Application à des données de l'étage subalpin des Alpes marifimes. I. L'analyse des donnćes floristiques. II. L'analyse des données écologiques ef l'analyse globale. Oecol. Plant., 6. 353-369 et 7, 125-146.

LE TACON F., NYS C., 1970. Les sols du Massif de Haye et leur influence sur le comportement des réserves de Hêtre en taillis-sous-futaie. Rev. forest. franç., 22, 5, 545-552.

MÉNARD M., BÉLANGER J., 1976. Etude des relations entre les caractères du milieu et la production forestière par l'analyse factorielle des correspondances. Serv, de la rech. Dir. gén. des for, min. des Ter. et For, du Québec, Mémoire n' 24, 74 p.

NUSSBAUM A., 1974. Contribution da la mise au point d'une méthode de cartographie des stations forestières. Application à la forêt de la Montagne (Meuse). Mémoire $3^{\circ}$ année E.N.I.T.E.F., 70 p. t annexes.

PARDÉ J., 1962. Table de production pour les forêts de Chêne rouvre de qualité tranchage du secteur ligérien. Notes fechniques forestières $n^{\circ} 11 ; 1$ re section, Station de Recherches ef Expériences forestières, Nancy, 6 p.

PICARD J. F., 1970. Les forêts sur Rhétien dans le département des Vosges. Nouvelle contribution à la mise au point d'une méthode dynamique d'éfude phyło-écologique du milieu forestier. Thèse $3^{\text {e }}$ cycle, Nancy, 74 p. + tableau.

PICARD J. F., 1976. La forêt domaniale de Bellême. Approche phyto-écologique. Document Laboratoire Phyto-écologie du C.N.R.F., 58 p. + annexe (sous presse).

POLGE H., KELLER R., 1970. Première appréciation de la qualité du bois en forêt par utilisation d'un torsiomètre. Ann. Sci. forest., 27, 2, 197-223.

POLGE H., 1973. Etat actuel des recherches sur la qualité du bois de Hêtre. Bull, tech. O.N.F., 4.

ROMANE F, 1972. Application à la phyto-écologie de quelques méthodes d'analyse multivariable, Thèse docteur-ingénieur, Université des Sciences et Techniques du Languedoc, Montpellier. 110 p.

SCHOBER R., 1967. Die Rotbuche. Sauerlander's Verlag, Frankfurt/Main, 333 p. 
TIMBAL J., 1973. La végétation de la forêt de Sainte-Hélène (Vosges). Exemple d'analyse floristicostatistique de phytocénose forestière. Vegetatio, 27, 4/6, 267-321.

TIMBAL J., 1974. Principaux caractères écologiques et floristiques des hêtraies du Nord-Est de la France. Ann. Sci. forest., 31, 1. 27-45.

VENET J., 1975. Le massif forestier de Haye. Document Syl. et Tech. $75-02$ de I'E.N.G.R.E.F., 86 p.

\section{ANNEXE \\ Liste des espèces ayant participé à l'analyse}

Le numéro précédant les noms permettent de renseigner les figures 1 et 2 . Le chiffre qui suit, entre parenthèses, est le nombre d'apparitions dans les sept-cent soixante-douze relevés. $A=$ strate arborescente; $\mathrm{G}=$ strates arbustive + herbacée.

4 Acer compestre L. G

5 Acer platanoides L. A

6 Acer platanoides L. G

7 Acer pseudoplatanus L. A

8 Acer pseudoplatanus L. G

10 Actaea spicato L.

11 Adoxa moschateilina (Tourn.) L.

12 Aegopodium podograria $\mathrm{L}$.

13 Agropyrum caninum (L.) P. B.

14 Ajuga reptans $L$.

15 Alliaria officinalis Andrz.

16 Allium ursinum $\mathrm{L}$.

18 Anemone nemorosa $\mathrm{L}$.

19 Anemone ronunculoides $\mathrm{L}$.

20 Angelica silvestris L.

21 Anthericum liliago L.

22 Anthericum ramosum L.

23 Anthriscus silvestris Hoffm.

24 Aquilegia vulgaris $\mathrm{L}$.

27 Arum maculatum L.

28 Asarum europoeum L.

29 Asperula odorato $\mathrm{L}$.

31 Athyrium filix femina (L.) Roth

32 Atrichum undulatum P. Beauv.

33 Afropa belladonna $L$.

34 Berberis vulgoris $\mathrm{L}$.

35 Befula verrucosa Ehrh. A

36 Befula verrucosa Ehrh. G

37 Brachypodium pinnatum (L.) B. P.

38 Brachypodium silvaticum $(H)$ RS.

39 Bromus osper Murr.

40 Calamintha officinalis $M$.

41 Campanula trachelium L.

42 Cardamine pratensis L.

43 Carex digitata L.

44 Carex giauca Murr.

45 Carex montana L.
46 Carex pairoei Hoppe

48 Carex silvatica Huds

49 Corpinus befulus L. A

50 Carpinus befulus L. G

51 Centaurea montana $\mathrm{L}$.

52 Cephalanthera pallens (J.) Rich.

53 Cephalanthera rubro (L.) Rich.

54 Circaea lutetiana $\mathrm{L}$.

55 Clematis vitalba $\mathrm{L}$.

56 Colchicum aufumnale L.

57 Convallaria maíalis L.

58 Cornus mas L.

59 Cornus sanguinea $\mathrm{L}$.

60 Corydallis cava (L.) Schw. K.

61 Corydallis solida (L.) Sm.

62 Corylus avellana $\mathrm{L}$.

63 Crataegus monogyna Jacq.

64 Crataegus oxyocantho L.

66 Dactylis glomerafo L.

67 Daphne laureola L.

68 Daphne mezereum L.

69 Dentaria pinnata $\mathrm{Lmk}$.

70 Deschampsia caespitosa (L.) PB

71 Dicranum scoparium Hedw.

72 Digitalis lutea L.

73 Elymus europoeus L.

74 Epilobium angustifolium L.

75 Epilobium montanum L.

77 Epipactis latifolia (L.) All.

78 Equisefum silvaticum L.

80 Eupatorium eu-cannabinum L.

82 Euphorbia cyparissias L.

83 Euphorbia dulcis L.

85 Eurhynchium striatum (Schreb) Sch.

87 Evanymus vulgaris Mill.

88 Fagus silvatica L. A

89 Fagus silvatica L. G 
90 Festuca gigantea (L.) Vill.

91 Fesfuca heierophylla Lmk.

92 Fesfuca silvatica Vill.

93 Ficaria verna Huds.

94 Fissidens faxifolius Hedw.

95 Fragaria vesca L.

96 Fraxinus excelsior L. A

97 Fraxinus exce/sior L. G

99 Galeopsis tefrahit L.

100 Golium aparine L.

101 Galium asperum Schreb.

102 Galium mollugo L.

103 Galium silvaticum L.

104 Genista pilosa L.

106 Geranium robertianum L.

107 Geum urbanum L.

108 Glechoma hederaceum L.

109 Hedera helix L.

110 Helleborus foetidus L.

111 Hepatica triloba Chaix

112 Heracleum sphondylium L.

113 Hierocium murorum L.

115 Hypericum hirsufum L.

116 Hypericum perforatum $\mathrm{L}$.

118 Lamium galeobdolon (L.) Cr.

119 Laserpitium latifolium L.

120 Lathyrus vernus (L.) Bernh.

121 Ligustrum vulgare L. G

122 Listera ovata (L.) R. Br.

124 Lithospermum purpureo coeruleum

125 Lonicera xylosteum L.

126 Luzula albida (Hoffm.) DC.

127 Luzula pilosa (L.) Will.

129 Melampyrum pratense L. P. P.

130 Melica nutans L.

131 Melica uniflora Retz

132 Meliffis melissophyllum L.

133 Mercurialis perennis $\mathrm{L}$.

Polygonatum mulfiflorum (L) All.

163 Polygonatum adoratum (Mili) P. F.

164 Polystichum filix mas (L.) Roth.

165 Polystichum spinulosum Lmk et Dc
(14) 166 Polytrichum formosum Hedw.

(239) 167 Populus tremula L. A

(9) 168 Populus tremula L. G

(30) 169 Pofentilla fragariastrum Ehrh.

(120) 170 Primula elatior (L.) Schreb.

(236) 171 Primula officinalis (L.) Hill.

(51) 172 Prunus avium L. A

(247) 173 Prunus avium L. G

(37) 174 Prunus spinosa $\mathrm{L}$.

(8) 175 Pleridium aquilinum (L.) Kuhn.

(7) 176 Pulmonaria officinalis $\mathrm{L}$.

(4) 177 Quercus lonuginosa Lmk. A

(324) 178 Quercus Ianuginosa Lmk. G

(2) 179 Quercus pedunculafa Ehrh. A

(16) 180 Quercus pedunculata Ehrh. G

(95) 181 Quercus sessilifiora Salisb. A

(24) 182 Quercus sessilifiora Salisb. G

(747) 184 Ranunculus auricomus L.

(52) 185 Ranunculus breynicus $\mathrm{Cr}$.

(380) 186 Rhamnus cathartica L

(156) 187 Rhamnus frangula $\mathrm{L}$.

(45) 189 Rhytidiadelphus triquefer $\left(\mathrm{H}_{.}\right)$Var

(35) 190 Ribes alpinum L.

(11) 191 Ribes rubrum L.

(504) 192 Ribes uva crispa L.

(23) 195 Rosa arvensis L.

(251) 196 Rubus coesius L.

(318) 197 Rubus idacus L.

(11) 198 Rubus saxatilis L.

(64) 199 Rubus sp.

200 Rubus ulmifolius Schott.

103) 205 Sambucus nigra L.

(292) 206 Sanicula europaea L.

(552) 208 Scilla bifolia L.

(28) 209 Scrofularia nodosa L.

(268) 210 Senecio fuchsii Gmel.

(249) 211 Sesleria coerulea (L.) Ard.

(11) 213 Solanum dulcamara L.

(149) 214 Solidago virga aurea L.

(25) 216 Sorbus aria (L.) Cr. G

(5) 217 Sorbus domestica L. A

(43) 219 Sorbus torminalis (L.) Cr. A

(190) 220 Sorbus torminalis (L.) Cr. G

221 Stellaria holosiea L.

(3) 223 Toraxacum officinale Webb.

(263) 226 Teucrium scorodonio $\mathrm{L}$.

(45) 228 Thuidium tamariscifolium (H.) Lind.

(27) 230 Tilia cordata Mill. G

(6) 231 Tilia platyphyllos Scop. A

(71) 232 Tilia platyphyllos Scop. G

(17) 233 Ulmus campestris L. A

(426) 234 Ulmus campestris L. G

(6) 236 Ulmus scraba Mill. G

(120) 237

Valeriana officinalis $\mathrm{L}$.

(215) 238 Veronica chamaedrys L.

(50) 239

(27) 240

Veronica montana $\mathrm{L}$.

(4) 241 Viburnum Iantano L.

Annales des Sciences lofestières. - 1979 
242 Viburnum opulus L.

243 Vicia sepium L.

244 Vinca minor L.

245 Vinceloxicum officinale $M$.

246 Viola hirta L.

247 Viola mirabilis L.

248 Viola silvestris (Lmk) Rchb.

251 Lonicera periclymenum L.

253 Maianthemum bifolium (L.) Schm.
(252) 254 Stachys officinalis (L.) Trevis

(358) 255 Stachys silvaticus L.

(64) 258 Campanula persicaefolio $\mathrm{L}$.

(2) 259 Carex pallescens $\mathrm{L}$.

(6) 260 Corex pilulifera $\mathrm{L}$.

(187) 261 Carex polyrrhiza Wallr.

(575) 265 Hypericum montanum L.

(21) 266 Urtica dioica L.

(2) 267 Molinia caerulea (L.) Moench
(5)

(22)

(2)

(2)

(1)

(1)

(1)

(3)

(4) 